\title{
Dynamical 1D models of passive piezoelectric sensors
}

\author{
Cédric Bellis and Sébastien Imperiale \\ Department of Applied Physics \& Applied Mathematics \\ Columbia University, New York NY, 10027
}

\begin{abstract}
This study concerns the mathematical modeling of anisotropic and transversely inhomogeneous slender piezoelectric bars. Such rod-like structures are employed as passive sensors aiming at measuring the displacement field on the boundary of an underlying elastic medium excited by an external source. Based on the coupled three-dimensional dynamical equations of piezoelectricity in the quasi-electrostratic approximation, a set of limit problems is derived using formal asymptotic expansions of the electric potential and elastic displacement fields. The nature of these problems depends strongly on the choice of boundary conditions, therefore an appropriate set of constrains is introduced in order to derive 1D models that are relevant to the measurement of a displacement field imposed at one end of the bar. The structure of the first-order electric and displacement fields as well as the associated coupled limit equations are determined. Moreover, the properties of the homogenized material parameters entering these equations are investigated in various configurations. The obtained 1D models of piezoelectric sensors are analyzed and it is finally shown how they enable the identification of the boundary displacement associated with the probed elastic medium.
\end{abstract}

\section{Introduction}

The transient wave-based imaging of elastic solids bear relevance to a wide range of applications such as nondestructive material testing, detection of buried objects, seismic imaging or inverse scattering problems. Tackling such inverse problems generally requires the knowledge of boundary data, provided by the measurements, that are over-determined, in the sense that both displacement and traction are known on the boundary, relative to what is normally necessary for solving the well-posed forward problem. The development of practical and robust algorithms based on full-waveform (or partial) measurements has been the subject of intense studies over the last decades. For instance, classical minimization-based approaches exploit the data through a misfit cost function, see e.g. [6, 20, 21, 23. Alternatively, the so-called qualitative methods are commonly centered on the development of an indicator function of scattering obstacles, see e.g. [3, 4, 5, 7, 9, 19,

Among the variety of available devices relevant to the measurement of boundary displacement, one is interested here in ultrasonic piezoelectric transducers. Such transducers are made of piezoelectric materials which have the property to convert mechanical energy into electrical energy, and reciprocally. Such devices are placed in contact with the probed elastic medium and they can be used as the sources of the illuminating elastic waves as well as, as the receivers of the associated echos [24, 26]. In this article, one focuses on this latter reception regime, or sensor mode, where a measurable electric charge or current is associated with an elastic displacement field itself generated by the mechanical waves impinging the sensor. It is further assumed that these waves are produced within the underlying solid by an external source which is not discussed. The piezoelectric phenomenon is investigated here within the framework of the quasi-static piezoelectric model [11, 12, which features the equations of elastodynamics coupled to Maxwell's equations reduced to a scalar electric potential. In this context, a number of issues arise in connection with the applications considered. On the one hand, in their most general form, the measurements are provided in the form of an integral operator (in time and space) acting on 
the elastodynamic state associated with the echoes recorded at the sensor's interface with the probed medium. On the other hand, only the time-depend and scalar electric field, rather than the vectorial displacement, is accessible. In other words, the mapping between boundary elastic field and measured electric potential lacks of injectivity, therefore, the available measurements significantly contribute to the ill-posedness of the inverse problem considered.

To deal with the aforementioned impediments, the ensuing analysis pursues an alternate route to the full $3 \mathrm{D}$ problem by considering a geometrical configuration where the transverse dimensions of the bar are small compared to the characteristic length-scales of the mechanical and electrical phenomena occurring within the sensor. Therefore, our purpose is to investigate the limit behavior of the displacement and electric fields as the diameter of the bar cross-section tends to zero. When taking into account only first-order contributions in the coupling with the underlying probed solid, i.e. when the bar is employed as a passive sensor, then the resulting dynamical 1D models of piezoelectric sensors yield simplified interpretations of the measured electric potential as a function of the mechanical source imposed at one extremity of the sensor.

Notably, some limit equivalent piezoelectric problems have been derived based on a number of approximations mainly motived by physical arguments: 1D propagation of plane waves in an infinite piezoelectric domain and $0 \mathrm{D}$ equivalent models such as the Mason circuit or the KLM model, see [25] for a discussion of such approximations. Alternatively, in this article, the sought 1D models are obtained by formal asymptotic expansions of the unknowns of the original 3D piezoelectric problem. This asymptotic approach is a classical method and one can refer to 8, 28, for a review on the asymptotic analysis procedure in the case of purely elastic rods and plates. This approach has been successfully employed to model thin piezoelectric plates, see e.g. [15, 16, 22, 27, and the references therein, and later on, static slender piezoelectric rods [17, 29]. In this approach it is well-known that the scaling of the unknowns and the assumption on the data, in particular on the imposed boundary conditions and the body forces, are essential in the resulting limit problem (as pointed out in [8] Sec. 1.10). For instance, taking into account tension-compression or torsion and flexion effects requires the assumption of asymptotically correct boundary conditions with respect to the featured small geometrical parameter. Moreover, in the derivation of limit dynamical models, these considerations also apply to the material parameters such as the mass density. In the case of purely elastic rods a comparison between the two different models obtained in [2] and [13], clearly shows how the assumed scaling of the mass density determines the resulting time-domain model.

In this study, we derive a number of limit dynamical $1 \mathrm{D}$ piezoelectric models assuming a proper set of boundary conditions, corresponding to the passive sensor configuration. In particular, no a priori scaling is introduced in this analysis but an appropriate boundary condition is imposed on the sensor's lateral surface in order to take into account transverse displacement effects. Moreover, we assume that the bar is anisotropic and transversely inhomogeneous. Following the introduction of the 3D piezoelectric mathematical model in Section 2, the formal asymptotic expansion approach is employed and the structure of the first-order elastic and electric fields is obtained in Section 3. The associated limit equations and homogenized material parameters are derived in Section 4 together with corresponding positivity and symmetry properties. Some simplifications occurring in various configurations are also discussed. Finally, the complete 1D models involving the asymptotic contributions of the boundary conditions are deduced. Section 5 concerns the comparison of the resulting limit problem with the classical Bernoulli-Navier rod model, as well as a discussion about the coupling with the probed solid and the identification of the corresponding boundary displacement.

\section{Mathematical model of piezoelectric bars}

\subsection{Preliminaries}

To derive the effective piezoelectric model, a family of problems posed in thin 3D piezoelectric domains, referred to as bars, are considered and they involve a small geometrical parameter $\delta>0$ associated with the transverse dimensions of the bars. Although a given piezoelectric bar is characterized by a given value of $\delta$, the effective model will be constructed by an asymptotic analysis as $\delta$ tends to zero. Therefore, it is considered that generic 

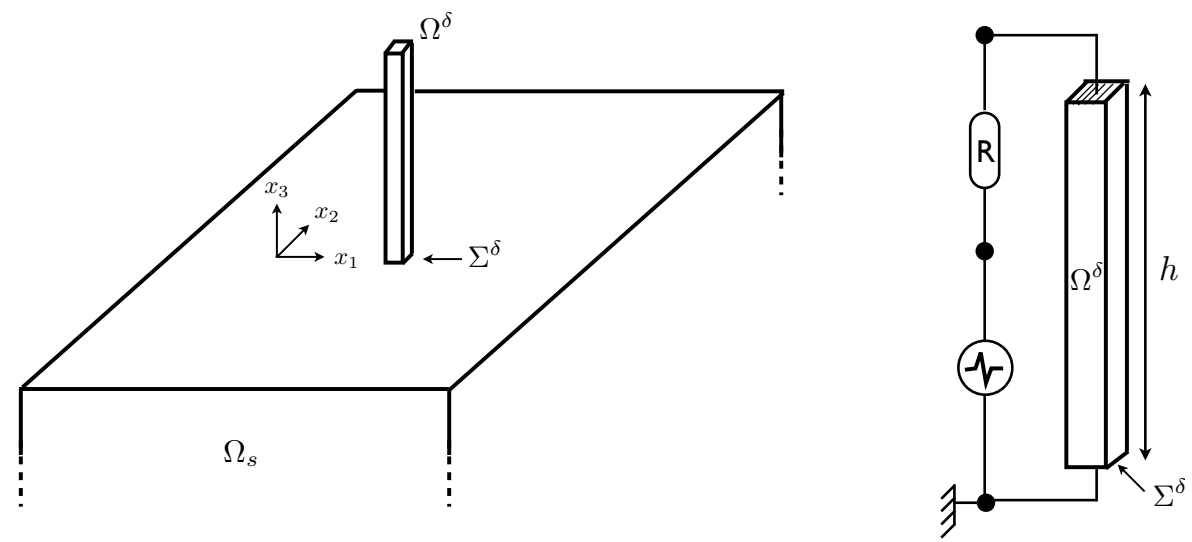

Figure 1: Piezoelectric sensor $\Omega^{\delta}$ and underlying elastic medium $\Omega_{s}$ (left) - Electrical connectivity between sensor and measurement device (right).

configurations are obtained by a uniform scaling of the transverse variables from a normalized reference domain. In particular, we consider a transversely normalized bar $\Omega$ defined as

$$
\Omega=S \times(0, h),
$$

with the cross-section $S$ being an open, normalized, connected, bounded and Lipschitz subset of $\mathbb{R}^{2}$. We also assume that $\partial \Omega$ is a smooth enough (Lipschitz) manifold along which the normal unit vector $\boldsymbol{n}$ can be defined.

Remark 2.1 Classically, the symbol "^" will be used to denote the variables corresponding to the normalized domain $\Omega$.

We consider thin domains parametrized by a strictly positive scalar $\delta$ and defined by

$$
\Omega^{\delta}=\mathcal{G}_{\delta}(\Omega)=\mathcal{G}_{\delta}(S) \times(0, h)=S^{\delta} \times(0, h),
$$

where $\mathcal{G}_{\delta}$ is the transformation $\mathcal{G}_{\delta}: \hat{\boldsymbol{x}}=\left(\hat{x}_{1}, \hat{x}_{2}, x_{3}\right) \mapsto \boldsymbol{x}=\left(x_{1}, x_{2}, x_{3}\right)=\left(\delta \hat{x}_{1}, \delta \hat{x}_{2}, x_{3}\right)$. In the sequel, we shall use systematically the transformation from $\Omega$ to $\Omega^{\delta}$ via the change of variables $\mathcal{G}_{\delta}$.

\subsubsection{Notations and algebraic properties}

The euclidean scalar product of $\mathbb{R}^{3}$ reads

$$
\boldsymbol{u} \cdot \boldsymbol{v}=\sum_{i=1}^{3} u_{i} v_{i} \quad \forall(\boldsymbol{u}, \boldsymbol{v}) \in \mathbb{R}^{3} \times \mathbb{R}^{3} .
$$

The space $\mathcal{L}\left(\mathbb{R}^{3}\right)$ of linear mappings from $\mathbb{R}^{3}$ into itself, whose elements are second-order tensors satisfying

$$
(\varepsilon \cdot \boldsymbol{u})_{i}=\sum_{j=1}^{3} \varepsilon_{i j} u_{j} \quad \forall \varepsilon=\left(\varepsilon_{i j}\right) \in \mathcal{L}\left(\mathbb{R}^{3}\right), \forall \boldsymbol{u} \in \mathbb{R}^{3},
$$

is equipped with the scalar product

$$
\boldsymbol{\sigma}: \boldsymbol{\varepsilon}=\sum_{i, j=1}^{3} \sigma_{i j} \varepsilon_{i j}, \quad \forall(\boldsymbol{\sigma}, \boldsymbol{\varepsilon}) \in \mathcal{L}\left(\mathbb{R}^{3}\right) \times \mathcal{L}\left(\mathbb{R}^{3}\right) .
$$


We denote by $\mathcal{L}_{\mathrm{sym}}\left(\mathbb{R}^{3}\right)$ the space of symmetric second-order tensors whose elements satisfy $\varepsilon_{i j}=\varepsilon_{j i}$. Moreover, let $\mathcal{L}^{2}\left(\mathbb{R}^{3}\right)$ denote the space of linear mappings from $\mathcal{L}\left(\mathbb{R}^{3}\right)$ into itself, in which any element is associated with a fourth-order tensor such as $\mathcal{C}=\left(\mathcal{C}_{i j k l}\right)$ satisfying

$$
(\mathcal{C}: \varepsilon)_{i j}=\sum_{k, l=1}^{3} \mathcal{C}_{i j k l} \varepsilon_{k l}, \quad \forall(\mathcal{C}, \varepsilon) \in \mathcal{L}^{2}\left(\mathbb{R}^{3}\right) \times \mathcal{L}\left(\mathbb{R}^{3}\right)
$$

again we denote by $\mathcal{L}_{\text {sym }}^{2}\left(\mathbb{R}^{3}\right)$ the space of fourth-order tensors whose elements have both major and minor symmetries, i.e. $\mathcal{C}_{i j k l}=\mathcal{C}_{k l i j}=\mathcal{C}_{j i k l}$. Next, let $\mathcal{L}\left(\mathbb{R}^{3}, \mathcal{L}_{\text {sym }}\left(\mathbb{R}^{3}\right)\right)$ denote the space of linear mappings from $\mathbb{R}^{3}$ into $\mathcal{L}_{\text {sym }}\left(\mathbb{R}^{3}\right)$, i.e. that transform vectors into second-order tensors. Such operators are associated with third-order tensors such as $\mathbf{d}=\left(\mathrm{d}_{k i j}\right)$ satisfying

$$
(\mathbf{d} \cdot \boldsymbol{u})_{i j}=\sum_{k=1}^{3} \mathrm{~d}_{k i j} u_{k} \quad \text { and } \quad \mathrm{d}_{k i j}=\mathrm{d}_{k j i} .
$$

The transposed tensor $\mathbf{d}^{\top}$, with respect to the inner products (1) and (2), is an element of the space $\mathcal{L}\left(\mathcal{L}_{\text {sym }}\left(\mathbb{R}^{3}\right), \mathbb{R}^{3}\right)$ of linear mappings from symmetric second-order tensors to vectors, and it is defined by

$$
\left(\mathbf{d}^{\top}: \varepsilon\right)_{k}=\sum_{i, j=1}^{3} \mathrm{~d}_{k i j} \varepsilon_{i j}
$$

To characterize the asymptotic behavior of the solution to the piezoelectric equations w.r.t. the transverse dimensions, i.e. when the scalar $\delta$ tends to 0 , we need to decompose the differential operators into partial derivatives acting on the transverse directions, scaled with $\delta$, from the partial derivatives corresponding to the longitudinal variable. On introducing a canonical basis $\left(\boldsymbol{e}_{i}\right)$ and given any scalar function $\varphi$, we decompose the gradient operator as

$$
\nabla \varphi=\nabla_{S} \varphi+e_{3} \partial_{x_{3}} \varphi \quad \text { where } \quad \nabla_{S} \varphi=\left(\begin{array}{c}
\partial_{\hat{x}_{1}} \varphi \\
\partial_{\hat{x}_{2}} \varphi \\
0
\end{array}\right)
$$

Similarly, given an arbitrary vector-valued function $\boldsymbol{u}=\left(u_{i}\right)$, then the symmetric part of its gradient, denoted as $\varepsilon[\boldsymbol{u}]$, can be decomposed according to

$$
\varepsilon[\boldsymbol{u}]=\varepsilon_{S}[\boldsymbol{u}]+\varepsilon_{3}[\boldsymbol{u}]
$$

where $\varepsilon_{S}[\boldsymbol{u}]$ and $\varepsilon_{3}[\boldsymbol{u}]$ are defined by the following matrix representations

$$
\varepsilon_{S}[\boldsymbol{u}]=\frac{1}{2}\left(\begin{array}{ccc}
2 \partial_{\hat{x}_{1}} u_{1} & \partial_{\hat{x}_{2}} u_{1}+\partial_{\hat{x}_{1}} u_{2} & \partial_{\hat{x}_{1}} u_{3} \\
\partial_{\hat{x}_{1}} u_{2}+\partial_{\hat{x}_{2}} u_{1} & 2 \partial_{\hat{x}_{2}} u_{2} & \partial_{\hat{x}_{2}} u_{3} \\
\partial_{\hat{x}_{1}} u_{3} & \partial_{\hat{x}_{2}} u_{3} & 0
\end{array}\right) \quad \text { and } \quad \varepsilon_{3}[\boldsymbol{u}]=\frac{1}{2}\left(\begin{array}{ccc}
0 & 0 & \partial_{x_{3}} u_{1} \\
0 & 0 & \partial_{x_{3}} u_{2} \\
\partial_{x_{3}} u_{1} & \partial_{x_{3}} u_{2} & 2 \partial_{x_{3}} u_{3}
\end{array}\right) \text {. }
$$

Accordingly, the scalar divergence operator div, which maps vector fields of $\mathbb{R}^{3}$ to $\mathbb{R}$, is decomposed into

$$
\operatorname{div} \boldsymbol{u}=\operatorname{div}_{S} \boldsymbol{u}+\operatorname{div}_{3} \boldsymbol{u} \quad \text { where } \quad \operatorname{div}_{S} \boldsymbol{u}=\partial_{\hat{x}_{1}} u_{1}+\partial_{\hat{x}_{2}} u_{2} \quad \text { and } \quad \operatorname{div}_{3} \boldsymbol{u}=\partial_{x_{3}} u_{3} .
$$

Also, the notation div is introduced for the vectorial divergence operator mapping the space $\mathcal{L}\left(\mathbb{R}^{3}\right)$ of tensor fields to $\mathbb{R}^{3}$. Let us recall that

$$
(\operatorname{div} \boldsymbol{\sigma})_{i}=\operatorname{div}\left(\boldsymbol{\sigma}_{i}\right)
$$


where $\boldsymbol{\sigma}_{i}$ denotes the $i$-th line vector of $\boldsymbol{\sigma}$, i.e. $\boldsymbol{\sigma}_{i}=\boldsymbol{\sigma}^{\top} \cdot \boldsymbol{e}_{i}$ with $\boldsymbol{e}_{i}$ an element of a basis of $\mathbb{R}^{3}$. Given a matrix-valued function $\boldsymbol{\sigma}=\left(\sigma_{i j}\right)$, then the vectorial divergence is also decomposed in

$$
\operatorname{div} \boldsymbol{\sigma}=\operatorname{div}_{S} \boldsymbol{\sigma}+\operatorname{div}_{3} \boldsymbol{\sigma} \quad \text { with } \quad \operatorname{div}_{S} \boldsymbol{\sigma}=\sum_{i=1}^{3} \sum_{j=1}^{2} \partial_{\hat{x}_{j}} \sigma_{i j} \boldsymbol{e}_{i} \quad \text { and } \quad \operatorname{div}_{3} \boldsymbol{\sigma}=\sum_{i=1}^{3} \partial_{x_{3}} \sigma_{i 3} \boldsymbol{e}_{i} .
$$

\subsubsection{Green's formulae}

To be employed in the ensuing analysis, the Green's formulae on $S$ with boundary $\partial S$, are presented hereafter for the reader's convenience. Given any symmetric second-order tensor $\boldsymbol{\epsilon}(\boldsymbol{x}) \in \mathcal{L}_{\text {sym }}\left(\mathbb{R}^{3}\right)$, then one has for $(\varphi, \psi) \in H^{1}(S) \times H^{1}(S)$

$$
\int_{S} \operatorname{div}_{S}\left(\boldsymbol{\epsilon} \cdot \nabla_{S} \varphi\right) \psi \mathrm{d} s=-\int_{S} \boldsymbol{\nabla}_{S} \varphi \cdot \boldsymbol{\epsilon} \cdot \nabla_{S} \psi \mathrm{d} s+\int_{\partial S} \boldsymbol{\epsilon} \cdot \nabla_{S} \varphi \cdot \boldsymbol{n} \psi \mathrm{d} \ell,
$$

where $\boldsymbol{n}$ is the unit outward normal on $\partial S$. Moreover, for any symmetric fourth-order tensor $\mathcal{C}(\boldsymbol{x}) \in \mathcal{L}_{\text {sym }}^{2}\left(\mathbb{R}^{3}\right)$ and $(\boldsymbol{u}, \boldsymbol{v}) \in H^{1}(S)^{3} \times H^{1}(S)^{3}$ the following identity holds

$$
\int_{S} \operatorname{div}_{S}\left(\mathcal{C}: \varepsilon_{S}[\boldsymbol{u}]\right) \cdot \boldsymbol{v} \mathrm{d} s=-\int_{S} \varepsilon_{S}[\boldsymbol{u}]: \mathcal{C}: \varepsilon_{S}[\boldsymbol{v}] \mathrm{d} s+\int_{\partial S} \mathcal{C}: \varepsilon_{S}[\boldsymbol{u}] \cdot \boldsymbol{n} \cdot \boldsymbol{v} \mathrm{d} \ell
$$

In the previous identities, the integrals along $\partial S$ must be understood in the sense of the duality product between $H^{-1 / 2}(\partial S)$ and $H^{1 / 2}(\partial S)$.

\subsection{Piezoelectric model}

The physical properties of the piezoelectric bars are characterized by a number of parameters which, by assumption, depend only on the transverse variables, namely the second-order permittivity tensor $\boldsymbol{\epsilon}^{\delta}\left(x_{1}, x_{2}\right)$, third-order piezoelectric tensor $\mathbf{d}^{\delta}\left(x_{1}, x_{2}\right)$, mass density $\rho^{\delta}\left(x_{1}, x_{2}\right)$ and fourth-order elastic tensor $\mathcal{C}^{\delta}\left(x_{1}, x_{2}\right)$. These material parameters satisfy the following usual boundedness, positiveness and symmetry properties

Hypothesis 2.1 We assume that, for all $\left(x_{1}, x_{2}\right) \in \delta S$

$$
\boldsymbol{\epsilon}^{\delta}\left(x_{1}, x_{2}\right) \in \mathcal{L}_{\text {sym }}\left(\mathbb{R}^{3}\right), \quad \mathbf{d}^{\delta}\left(x_{1}, x_{2}\right) \in \mathcal{L}\left(\mathbb{R}^{3}, \mathcal{L}_{\text {sym }}\left(\mathbb{R}^{3}\right)\right), \quad \rho^{\delta}\left(x_{1}, x_{2}\right) \in \mathbb{R}, \quad \mathcal{C}^{\delta}\left(x_{1}, x_{2}\right) \in \mathcal{L}_{\text {sym }}^{2}\left(\mathbb{R}^{3}\right)
$$

and there exist a number of scalars $\epsilon_{ \pm}, \rho_{ \pm}$and $c_{ \pm}$such that

$$
\begin{gathered}
0<\epsilon_{-}|\boldsymbol{\psi}|^{2} \leq \boldsymbol{\psi} \cdot \boldsymbol{\epsilon}^{\delta}\left(x_{1}, x_{2}\right) \cdot \boldsymbol{\psi} \leq \epsilon_{+}|\boldsymbol{\psi}|^{2}, \\
0<\rho_{-} \leq \rho^{\delta}\left(x_{1}, x_{2}\right) \leq \rho_{+}, \quad 0<c_{-}|\varepsilon|^{2} \leq \varepsilon: \mathcal{C}^{\delta}\left(x_{1}, x_{2}\right): \varepsilon \leq c_{+}|\varepsilon|^{2} .
\end{gathered}
$$

for all $\boldsymbol{\psi} \in \mathbb{R}^{3}$ and $\varepsilon \in \mathcal{L}_{\text {sym }}\left(\mathbb{R}^{3}\right)$.

Note that this symmetry property of the piezoelectric tensor $\mathbf{d}$ entails the following identity

$$
\psi \cdot \mathbf{d}^{\delta^{\top}}: \varepsilon=\mathbf{d}^{\delta} \cdot \psi: \varepsilon
$$

Finally, without loss of generality the coordinate system is chosen so that $\boldsymbol{e}_{3}$ is a principal axis of inertia for the bar, i.e. such that one has

$$
\int_{\delta S} \rho^{\delta} \boldsymbol{x} \cdot \boldsymbol{e}_{1} \mathrm{~d} \boldsymbol{x}=0 \quad \text { and } \quad \int_{\delta S} \rho^{\delta} \boldsymbol{x} \cdot \boldsymbol{e}_{2} \mathrm{~d} \boldsymbol{x}=0 .
$$


The time-domain coupled equations governing the electric potential $\varphi^{\delta}$ and the elastic displacement $\boldsymbol{u}^{\delta}$ in the piezoelectric bar $\Omega^{\delta}$ considered are

$$
\begin{cases}\operatorname{div}\left(\boldsymbol{\epsilon}^{\delta} \cdot \boldsymbol{\nabla} \varphi^{\delta}-\mathbf{d}^{\delta^{\top}}: \boldsymbol{\varepsilon}\left[\boldsymbol{u}^{\delta}\right]\right)=0, & \boldsymbol{x} \in \Omega^{\delta}, t>0, \\ \rho^{\delta} \partial_{t}^{2} \boldsymbol{u}^{\delta}-\operatorname{div}\left(\mathcal{C}^{\delta}: \varepsilon\left[\boldsymbol{u}^{\delta}\right]+\mathbf{d}^{\delta} \cdot \boldsymbol{\nabla} \varphi^{\delta}\right)=\mathbf{0}, & \boldsymbol{x} \in \Omega^{\delta}, t>0 .\end{cases}
$$

This system of field equations is completed by a set of boundary conditions. First, the following electricdisplacement-free condition is assumed on the lateral surface.

$$
\left(\boldsymbol{\epsilon}^{\delta} \cdot \nabla \varphi^{\delta}-\mathbf{d}^{\delta^{\top}}: \varepsilon\left[\boldsymbol{u}^{\delta}\right]\right) \cdot \boldsymbol{n}=0, \quad \boldsymbol{x} \in \partial S^{\delta} \times(0, h), \quad t>0
$$

This assumption is a valid approximation when a high permittivity contrast occurs between the piezoelectric bar and the surrounding media (see [12] for more details). Moreover, to write the elastic boundary conditions, one introduces a partition of $\partial S^{\delta}$ as

$$
\overline{\partial S^{\delta}}=\overline{\Gamma_{0}^{\delta}} \cup \overline{\Gamma_{N}^{\delta}}
$$

such that $\Gamma_{0}^{\delta} \cap \Gamma_{N}^{\delta}=\emptyset$ and where $\Gamma_{N}^{\delta}$ is associated with the homogeneous elastic Neumann condition

$$
\left(\mathcal{C}^{\delta}: \varepsilon\left[\boldsymbol{u}^{\delta}\right]+\mathbf{d}^{\delta} \cdot \nabla \varphi^{\delta}\right) \cdot \boldsymbol{n}=\mathbf{0}, \quad \boldsymbol{x} \in \Gamma_{N}^{\delta} \times(0, h), \quad t>0
$$

whereas $\Gamma_{0}^{\delta}$, which may vanish, corresponds to the following homogeneous Neumann and Dirichlet elastic conditions

$$
\left(\boldsymbol{I}-\boldsymbol{e}_{3} \otimes \boldsymbol{e}_{3}\right) \cdot\left[\left(\mathcal{C}^{\delta}: \varepsilon\left[\boldsymbol{u}^{\delta}\right]+\mathbf{d}^{\delta} \cdot \boldsymbol{\nabla} \varphi^{\delta}\right) \cdot \boldsymbol{n}\right]=\mathbf{0} \quad \text { and } \quad \boldsymbol{u} \cdot \boldsymbol{e}_{3}=0, \quad \boldsymbol{x} \in \Gamma_{0}^{\delta} \times(0, h), \quad t>0,
$$

where $\boldsymbol{I}$ is the second-order identity tensor. This mixed elastic condition is intended to lead to a first-order dynamical model involving non-zero transverse displacements when $\left|\Gamma_{0}^{\delta}\right| \neq 0$.

At $x_{3}=0$, we consider that the displacement is imposed as

$$
\boldsymbol{u}_{b}^{\delta}\left(x_{3}=0\right)=\boldsymbol{t}_{b}^{\delta}, \quad\left(x_{1}, x_{2}\right) \in S^{\delta}, \quad t>0,
$$

where $\boldsymbol{t}_{b}^{\delta}$ is the elastic source term considered in this study. This mechanical excitation is generated by the echoes recorded by the sensor. We assume that the latter satisfies

$$
\boldsymbol{t}_{b}^{\delta}(\boldsymbol{x}, t)=\boldsymbol{t}_{b}(t)+r_{b}(t) \delta^{-1}\left(x_{2} \boldsymbol{e}_{1}-x_{1} \boldsymbol{e}_{2}\right)
$$

where $\boldsymbol{t}_{b}$ and $r_{b}(t)$ are constant terms with respect to $\boldsymbol{x}$. The reason to assume such a source term arises from the study of the coupling with the underlying medium, which will be discussed in Section 5 . At $x_{3}=h$, leeway is allowed in choosing the elastic constrain, therefore we consider hereafter the following clamped boundary condition

$$
\boldsymbol{u}^{\delta}\left(x_{3}=h\right)=\mathbf{0}, \quad\left(x_{1}, x_{2}\right) \in S^{\delta}, \quad t>0 .
$$

Remark 2.2 We could have employed here, the following traction-free condition

$$
\left(\mathcal{C}^{\delta}: \varepsilon\left[\boldsymbol{u}^{\delta}\right]+\mathbf{d}^{\delta} \cdot \nabla \varphi^{\delta}\right) \cdot \boldsymbol{n}=\mathbf{0}, \quad \boldsymbol{x} \in S^{\delta} \times\{h\}, \quad t>0 .
$$

However, we will show in the ensuing analysis (see Section 4.3.2) that this condition leads to a different 1D model for which the interpretation of the measured potential, as a function of the imposed elastic displacement at $x_{3}=0$, is much more involved. For this reason in connection with the intended application to inverse problems such model is dismissed from the present study. 
Next, at $x_{3}=0$ we assume that the bar is electrically connected to the ground which is mathematically modeled by imposing a homogeneous boundary condition on the electric potential, i.e.

$$
\varphi^{\delta}\left(x_{3}=0\right)=0, \quad\left(x_{1}, x_{2}\right) \in S^{\delta}, t>0
$$

At $x_{3}=h$ the piezoelectric bar is connected to a resistive measurement device (see Figure 1) which internal resistance $R$ is supposed to be scalable according to $R=\delta^{-p} R_{0}$ with, e.g., $p \in\{0,1,2\}$. This setting yields a measurement of the electric potential associated to the following mixed boundary condition (see [12])

$$
\varphi^{\delta}\left(x_{3}=h\right)=-\delta^{-p} R_{0} \frac{d}{d t} \int_{S^{\delta}, x_{3}=h}\left(\boldsymbol{\epsilon}^{\delta} \cdot \nabla \varphi^{\delta}-\mathbf{d}^{\delta^{\top}}: \boldsymbol{\varepsilon}\left[\boldsymbol{u}^{\delta}\right]\right) \cdot \boldsymbol{n} \mathrm{d} s, \quad t>0 .
$$

Note that in the case where the piezoelectric bar is used as an actuator, then a generator is employed to apply an electric potential at $x_{3}=h$, therefore, an electric source term $V(t)$ has to be added to the previous relation and $R$ can be interpreted as the internal resistance of the generator.

Finally we assume that the system is at rest at the initial time, i.e.

$$
\boldsymbol{u}^{\delta}(t=0)=\mathbf{0}, \quad \partial_{t} \boldsymbol{u}^{\delta}(t=0)=\mathbf{0} \quad \text { and } \quad \varphi^{\delta}(t=0)=0, \quad \boldsymbol{x} \in \Omega^{\delta} .
$$

In this article, our aim is to describe the behavior of the electric potential $\varphi^{\delta}$ and elastic displacement $\boldsymbol{u}^{\delta}$ arising in the piezoelectric bar $\Omega^{\delta}$ when the geometrical parameter $\delta$ tends to 0 . Therefore, it is useful to apply the change of variables $\mathcal{G}_{\delta}$ in order to work in the fixed, normalized geometry. Doing so, the parameter $\delta$ will appear as a coefficient into the governing equations of the problem. Thus, we introduce the fields $(\varphi, \boldsymbol{u})$ defined on $\Omega$ according to

$$
\varphi^{\delta}=\varphi \circ \mathcal{G}_{\delta}^{-1} \quad \text { and } \quad \boldsymbol{u}^{\delta}=\boldsymbol{u} \circ \mathcal{G}_{\delta}^{-1} .
$$

Moreover, we assume that the physical parameters can be transposed on the reference domain $\Omega$ according to the change of variables

$$
\boldsymbol{\epsilon}^{\delta}=\boldsymbol{\epsilon} \circ \mathcal{G}_{\delta}^{-1}, \quad \mathbf{d}^{\delta}=\mathbf{d} \circ \mathcal{G}_{\delta}^{-1}, \quad \rho^{\delta}=\rho \circ \mathcal{G}_{\delta}^{-1}, \quad \mathcal{C}^{\delta}=\mathcal{C} \circ \mathcal{G}_{\delta}^{-1}
$$

and the hypothesis 2.1 is naturally extended to $(\boldsymbol{\epsilon}, \mathbf{d}, \rho, \mathcal{C})$. The above definitions of the unknowns and parameters from their counterparts defined on the domain $\Omega^{\delta}$ using only a change of coordinates and not a scaling in the parameter $\delta$ appears to be a natural choice in the application to inverse problems considered in the article. However, in the literature concerning the justification of plate and rod models from three-dimensional linearized elasticity by asymptotic analysis it is commonly assumed that the unknowns, material parameters and loads are adequately scaled according to $\delta$. See [28] for a detailed description of the asymptotic analysis procedure applied to elastic rods, and [8] for a discussion on common scalings. It will appear in our analysis that an ansatz of the asymptotic expansion of $(\varphi, \boldsymbol{u})$ enables to derive a relevant 1D model, and that an a priori scaling of this solution is not necessary, yet could have simplified the calculation in a static configuration.

As pointed out in, e.g., [8] in the case of plates, the standard scaling of the sources is necessary to obtain meaningful models that take into account tension-compression versus torsion and flexion effects. Such an asymptotic procedure has been applied in [17] to derive a static piezoelectric rod model of Bernoulli-Navier type. The problem considered here differs in nature in that it does not involve any body force or imposed boundary traction, hence making such scaling irrelevant.

Finally, in order to model first-order inertia effects, the parameter $\rho$ is also commonly scaled according to $\delta$ (see e.g. [28, 8]) or equivalently a change of time variable is done $(t \leftarrow \delta t)$ to emphasize long time behavior. In our application, such a scaling or change of variable is not justified, then the assumed definition $\rho^{\delta}=\rho \circ \mathcal{G}_{\delta}^{-1}$ appears to be a natural choice. A detailed discussion regarding the resulting dynamical model, which is not of Bernoulli-Navier type, is provided in Section 5 . 
Using equation (6) and the decompositions (34) we find that $(\varphi, \boldsymbol{u})$ satisfy for $\hat{\boldsymbol{x}} \in \Omega$ and $t>0$

$$
\left\{\begin{array}{c}
\operatorname{div}_{S}\left(\boldsymbol{\epsilon} \cdot \nabla_{S} \varphi-\mathbf{d}^{\top}: \varepsilon_{S}[\boldsymbol{u}]\right)+\delta \operatorname{div}_{S}\left(\boldsymbol{\epsilon} \cdot \boldsymbol{e}_{3} \partial_{x_{3}} \varphi-\mathbf{d}^{\top}: \varepsilon_{3}[\boldsymbol{u}]\right) \\
+\delta \operatorname{div}_{3}\left(\boldsymbol{\epsilon} \cdot \nabla_{S} \varphi-\mathbf{d}^{\top}: \varepsilon_{S}[\boldsymbol{u}]\right)+\delta^{2} \operatorname{div}_{3}\left(\boldsymbol{\epsilon} \cdot \boldsymbol{e}_{3} \partial_{x_{3}} \varphi-\mathbf{d}^{\top}: \varepsilon_{3}[\boldsymbol{u}]\right)=0, \\
\delta^{2} \rho \partial_{t}^{2} \boldsymbol{u}-\operatorname{div}_{S}\left(\mathcal{C}: \varepsilon_{S}[\boldsymbol{u}]+\mathbf{d} \cdot \nabla_{S} \varphi\right)-\delta \operatorname{div}_{S}\left(\mathcal{C}: \varepsilon_{3}[\boldsymbol{u}]+\mathbf{d} \cdot \boldsymbol{e}_{3} \partial_{x_{3}} \varphi\right) \\
\quad-\delta \operatorname{div}_{3}\left(\mathcal{C}: \boldsymbol{\varepsilon}_{S}[\boldsymbol{u}]+\mathbf{d} \cdot \nabla_{S} \varphi\right)-\delta^{2} \operatorname{div}_{3}\left(\mathcal{C}: \varepsilon_{3}[\boldsymbol{u}]+\mathbf{d} \cdot \boldsymbol{e}_{3} \partial_{x_{3}} \varphi\right)=\mathbf{0} .
\end{array}\right.
$$

The lateral boundary conditions for the unknowns $(\varphi, \boldsymbol{u})$ are deduced from $(7,9)$ and they read for $t>0$

$$
\begin{cases}\left(\boldsymbol{\epsilon} \cdot \nabla_{S} \varphi-\mathbf{d}^{\top}: \varepsilon_{S}[\boldsymbol{u}]\right) \cdot \boldsymbol{n}+\delta\left(\boldsymbol{\epsilon} \cdot \boldsymbol{e}_{3} \partial_{x_{3}} \varphi-\mathbf{d}^{\top}: \varepsilon_{3}[\boldsymbol{u}]\right) \cdot \boldsymbol{n}=0, & \hat{\boldsymbol{x}} \in \partial S \times(0, h), \\ \left(\mathcal{C}: \boldsymbol{\varepsilon}_{S}[\boldsymbol{u}]+\mathbf{d} \cdot \nabla_{S} \varphi\right) \cdot \boldsymbol{n}+\delta\left(\mathcal{C}: \varepsilon_{3}[\boldsymbol{u}]+\mathbf{d} \cdot \boldsymbol{e}_{3} \partial_{x_{3}} \varphi\right) \cdot \boldsymbol{n}=\mathbf{0}, & \hat{\boldsymbol{x}} \in \Gamma_{N} \times(0, h), \\ \left(\boldsymbol{I}-\boldsymbol{e}_{3} \otimes \boldsymbol{e}_{3}\right) \cdot\left[\left(\mathcal{C}: \boldsymbol{\varepsilon}_{S}[\boldsymbol{u}]+\mathbf{d} \cdot \boldsymbol{\nabla}_{S} \varphi\right) \cdot \boldsymbol{n}+\delta\left(\mathcal{C}: \varepsilon_{3}[\boldsymbol{u}]+\mathbf{d} \cdot \boldsymbol{e}_{3} \partial_{x_{3}} \varphi\right) \cdot \boldsymbol{n}\right]=0, & \hat{\boldsymbol{x}} \in \Gamma_{0} \times(0, h), \\ \boldsymbol{u} \cdot \boldsymbol{e}_{3}=\mathbf{0} & \hat{\boldsymbol{x}} \in \Gamma_{0} \times(0, h),\end{cases}
$$

and finally the initial conditions are deduced from 14 as

$$
\boldsymbol{u}(t=0)=\mathbf{0}, \quad \partial_{t} \boldsymbol{u}(t=0)=\mathbf{0} \quad \text { and } \quad \varphi(t=0)=0 \quad \hat{\boldsymbol{x}} \in \Omega .
$$

Remark 2.3 Note that, even if the fields $\varphi$ and $\boldsymbol{u}$ are expressed in terms of the normalized coordinates from the change of variables (15), they still depend on the small parameter $\delta$ as they are solution of a piezoelectric problem which features $\delta$. The coupled problem satisfied by $(\varphi, \boldsymbol{u})$ is written hereafter.

\section{Formal asymptotic expansion approach}

We seek a regular asymptotic expansion in powers of $\delta$ of the unkowns, namely we assume that the solution $(\varphi, \boldsymbol{u})$ to $16,17,18)$ can be written in terms of a formal power series expansion in $\delta$ as

$$
\varphi=\varphi_{0}+\delta \varphi_{1}+\delta^{2} \varphi_{2}+\ldots \quad \text { and } \boldsymbol{u}=\boldsymbol{u}_{0}+\delta \boldsymbol{u}_{1}+\delta^{2} \boldsymbol{u}_{2}+\ldots
$$

where the fields $\varphi_{i}$ and $\boldsymbol{u}_{i}$ are solutions of coupled problems independent of $\delta$.

In the ensuing analysis, we adopt the standard methodology which consists in inserting (19) in (16) and identifying the series term by term in power of $\delta$, starting with the $\mathcal{O}(1)$ terms.

\subsection{Piezoelectric problem defined on 2D cross-section}

In this section we present a simple extension to the piezoelectric problem defined over the cross-section $S$ of a classical result concerning the elasticity problem likewise written on a $2 D$ geometry. To do so, let us introduce the space

$$
\mathcal{E}_{S}=\left\{\boldsymbol{w} \in H^{1}(S)^{3} \text { such that } \boldsymbol{\varepsilon}_{S}[\boldsymbol{w}]=\mathbf{0} \text { in } S\right\},
$$

with the associated subspace of functions whose third component vanishes on $\Gamma_{0}$

$$
\mathcal{E}_{S, 0}=\left\{\boldsymbol{w} \in \mathcal{E}_{S} \text { such that } \boldsymbol{w} \cdot \boldsymbol{e}_{3}=0 \text { on } \Gamma_{0}\right\} .
$$

A main characterization of $\mathcal{E}_{S}$ and $\mathcal{E}_{S, 0}$ is given by the following proposition (see, e.g., [18]) 
Proposition 3.1 The space $\mathcal{E}_{S}$ is of dimension 4 and satisfies

$$
\mathcal{E}_{S}=\operatorname{span}\left\{\boldsymbol{e}_{1}, \boldsymbol{e}_{2}, \boldsymbol{e}_{3}, \boldsymbol{m}\left(\hat{x}_{1}, \hat{x}_{2}\right)\right\}
$$

with the function $\boldsymbol{m}$ defined on $S$ as

$$
\boldsymbol{m}\left(\hat{x}_{1}, \hat{x}_{2}\right)=e_{3} \times \hat{\boldsymbol{x}}=\boldsymbol{e}_{2} \hat{x}_{1}-\boldsymbol{e}_{1} \hat{x}_{2} .
$$

Moreover, if $\left|\Gamma_{0}\right|=0$ then $\mathcal{E}_{S, 0}=\mathcal{E}_{S}$, whereas if $\left|\Gamma_{0}\right| \neq 0$ then $\mathcal{E}_{S, 0}$ is of dimension 3 and

$$
\mathcal{E}_{S, 0}=\operatorname{span}\left\{\boldsymbol{e}_{1}, \boldsymbol{e}_{2}, \boldsymbol{m}\left(\hat{x}_{1}, \hat{x}_{2}\right)\right\} .
$$

To facilitate the ensuing discussion, one can introduce a parameter $d \in \mathbb{N}$ that will conveniently be related to the dimension of the space $\mathcal{E}_{S, 0}$

Definition 3.1 Let $d=\operatorname{dim}\left(\mathcal{E}_{S, 0}\right)-1$, i.e. $d=3$ when $\left|\Gamma_{0}\right|=0$ or $d=2$ if $\left|\Gamma_{0}\right| \neq 0$.

Now let

$$
\left(f_{\varphi}, \boldsymbol{f}_{\boldsymbol{u}}\right) \in H^{-1}(S) \times H^{-1}(S)^{3} \text { and }\left(g_{\varphi}, \boldsymbol{g}_{\boldsymbol{u}}\right) \in H^{-\frac{1}{2}}(\partial S) \times H^{-\frac{1}{2}}\left(\Gamma_{N}\right)^{3} \text { with } \boldsymbol{g}_{\boldsymbol{u}} \cdot \boldsymbol{e}_{3}=0 \text { on } \Gamma_{0}
$$

and consider the system of field equations satisfied by $(\psi, \boldsymbol{v})$

$$
\begin{cases}\operatorname{div}_{S}\left(\boldsymbol{\epsilon} \cdot \nabla_{S} \psi-\mathbf{d}^{\top}: \varepsilon_{S}[\boldsymbol{v}]\right)=f_{\varphi}, & \text { in } S, \\ \operatorname{div}_{S}\left(\mathcal{C}: \varepsilon_{S}[\boldsymbol{v}]+\mathbf{d} \cdot \nabla_{S} \psi\right)=\boldsymbol{f}_{\boldsymbol{u}}, & \text { in } S,\end{cases}
$$

with associated boundary conditions

$$
\begin{cases}\left(\boldsymbol{\epsilon} \cdot \nabla_{S} \psi-\mathbf{d}^{\top}: \boldsymbol{\varepsilon}_{S}[\boldsymbol{v}]\right) \cdot \boldsymbol{n}=g_{\varphi} & \text { on } \partial S, \\ \left(\mathcal{C}: \boldsymbol{\varepsilon}_{S}[\boldsymbol{v}]+\mathbf{d} \cdot \boldsymbol{\nabla}_{S} \psi\right) \cdot \boldsymbol{n}=\boldsymbol{g}_{\boldsymbol{u}} & \text { on } \Gamma_{N}, \\ \left(\boldsymbol{I}-\boldsymbol{e}_{3} \otimes \boldsymbol{e}_{3}\right) \cdot\left[\left(\mathcal{C}: \boldsymbol{\varepsilon}_{S}[\boldsymbol{v}]+\mathbf{d} \cdot \boldsymbol{\nabla}_{S} \psi\right) \cdot \boldsymbol{n}\right]=\boldsymbol{g}_{\boldsymbol{u}} & \text { on } \Gamma_{0}, \\ \boldsymbol{v} \cdot \boldsymbol{e}_{3}=0 & \text { on } \Gamma_{0} .\end{cases}
$$

Then on can prove the following proposition where the space $\mathcal{E}_{S, 0}$ plays a fundamental role:

Proposition 3.2 There exists a solution

$$
(\psi, \boldsymbol{v}) \in H^{1}(S) \times\left\{\boldsymbol{w} \in H^{1}(S)^{3} \text { such that } \boldsymbol{w} \cdot \boldsymbol{e}_{3}=0 \text { on } \Gamma_{0}\right\}
$$

to the problem 20 21] if the so-called compatibility conditions are met,

$$
\begin{aligned}
& \int_{S} f_{\varphi} d \hat{s}=\int_{\partial S} g_{\varphi} d \hat{\ell} \\
& \int_{S} \boldsymbol{f}_{\boldsymbol{u}} \cdot \boldsymbol{w} d \hat{s}=\int_{\partial S} \boldsymbol{g}_{\boldsymbol{u}} \cdot \boldsymbol{w} d \hat{\ell} \quad \text { for all } \boldsymbol{w} \in \mathcal{E}_{S, 0} .
\end{aligned}
$$

Moreover, the solution $(\psi, \boldsymbol{v})$ is unique up to an additive constant over $S$ for $\psi$ and up to an element of $\mathcal{E}_{S, 0}$ for $\boldsymbol{v}$.

It is noticeable that, if $\left|\Gamma_{0}\right| \neq 0$ (resp. $\left|\Gamma_{0}\right|=0$ ) the space $\mathcal{E}_{S, 0}$ is of dimension 3 (resp. 4) then (22) constitutes a set of 4 (resp. 5) equations. 


\subsection{Structure of limit displacement and electric fields.}

From $(16)$, the identification of $\mathcal{O}(1)$ terms yields

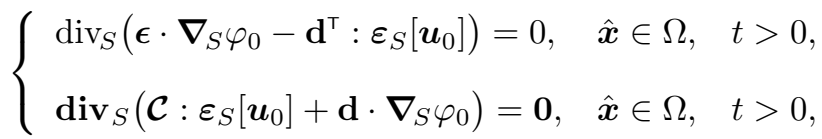

and from (17) we deduce the corresponding boundary conditions as

$$
\begin{cases}\left(\boldsymbol{\epsilon} \cdot \nabla_{S} \varphi_{0}-\mathbf{d}^{\top}: \boldsymbol{\varepsilon}_{S}\left[\boldsymbol{u}_{0}\right]\right) \cdot \boldsymbol{n}=0, & \hat{\boldsymbol{x}} \in \partial S \times(0, h), \quad t>0, \\ \left(\mathcal{C}: \boldsymbol{\varepsilon}_{S}\left[\boldsymbol{u}_{0}\right]+\mathbf{d} \cdot \boldsymbol{\nabla}_{S} \varphi_{0}\right) \cdot \boldsymbol{n}=\mathbf{0}, & \hat{\boldsymbol{x}} \in \Gamma_{N} \times(0, h), \quad t>0, \\ \left(\boldsymbol{I}-\boldsymbol{e}_{3} \otimes \boldsymbol{e}_{3}\right) \cdot\left[\left(\mathcal{C}: \boldsymbol{\varepsilon}_{S}\left[\boldsymbol{u}_{0}\right]+\mathbf{d} \cdot \boldsymbol{\nabla}_{S} \varphi_{0}\right) \cdot \boldsymbol{n}\right]=\mathbf{0}, & \hat{\boldsymbol{x}} \in \Gamma_{0} \times(0, h), \quad t>0, \\ \boldsymbol{u}_{0} \cdot \boldsymbol{e}_{3}=0, & \hat{\boldsymbol{x}} \in \Gamma_{0} \times(0, h), \quad t>0 .\end{cases}
$$

From Proposition 3.2 we deduce that, for each $t>0$ and $x_{3} \in(0, h), \varphi_{0}$ is zero up to a constant and $\boldsymbol{u}_{0}$ is zero up to an element in $\mathcal{E}_{S, 0}$, so we can write

$$
\varphi_{0}(\hat{\boldsymbol{x}}, t) \equiv \phi_{0}\left(x_{3}, t\right) \text { and } \boldsymbol{u}_{0}(\hat{\boldsymbol{x}}, t) \equiv \boldsymbol{t}_{0}\left(x_{3}, t\right)+\boldsymbol{m}\left(\hat{x}_{1}, \hat{x}_{2}\right) r_{0}\left(x_{3}, t\right)
$$

which is defined in terms of scalar functions $r_{0}\left(x_{3}, t\right)$ as well as $t_{0, i}\left(x_{3}, t\right)$, for $i=1, \ldots, d$, such that $\boldsymbol{t}_{0}$ reads

$$
\boldsymbol{t}_{0}\left(x_{3}, t\right)=\sum_{i=1}^{d} t_{0, i}\left(x_{3}, t\right) \boldsymbol{e}_{i}
$$

The structure of the solution given by the equations $\sqrt{23}$ is extended in the entire domain $\bar{\Omega}$, i.e. including the boundaries at $x_{3}=0$ and $h$. It will appear in the following that such a choice is compatible with the boundary conditions featured in (17) when $\boldsymbol{t}_{b}$ is appropriately defined (see Section 4.3.1). However in full generality, boundary layers may exist at the extremities of the bars implying the necessity to modify the initial ansatz (19) or to introduce adapted correctors (see, for instance, [10] for plates or [1] in the periodic homogenization theory).

\subsection{Definition of canonical problems}

The identification of $\mathcal{O}(\delta)$ terms in 16 entails

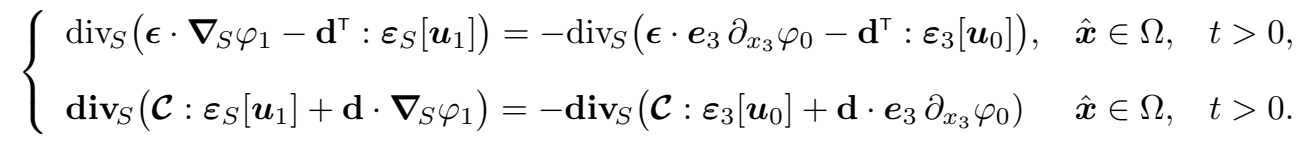

These equations are completed by boundary conditions on $\partial S \times(0, h)$ by identifying the $\mathcal{O}(\delta)$ terms in the equations (17):

$$
\left\{\begin{array}{lll}
\left(\boldsymbol{\epsilon} \cdot \nabla_{S} \varphi_{1}-\mathbf{d}^{\top}: \boldsymbol{\varepsilon}_{S}\left[\boldsymbol{u}_{1}\right]\right) \cdot \boldsymbol{n}=-\left(\boldsymbol{\epsilon} \cdot \boldsymbol{e}_{3} \partial_{x_{3}} \varphi_{0}-\mathbf{d}^{\top}: \varepsilon_{3}\left[\boldsymbol{u}_{0}\right]\right) \cdot \boldsymbol{n}, & \hat{\boldsymbol{x}} \in \partial S \times(0, h), & t>0, \\
\left(\mathcal{C}: \boldsymbol{\varepsilon}_{S}\left[\boldsymbol{u}_{1}\right]+\mathbf{d} \cdot \boldsymbol{\nabla}_{S} \varphi_{1}\right) \cdot \boldsymbol{n}=-\left(\mathcal{C}: \boldsymbol{\varepsilon}_{3}\left[\boldsymbol{u}_{0}\right]+\mathbf{d} \cdot \boldsymbol{e}_{3} \partial_{x_{3}} \varphi_{0}\right) \cdot \boldsymbol{n} & \hat{\boldsymbol{x}} \in \Gamma_{N} \times(0, h), & t>0, \\
\left(\boldsymbol{I}-\boldsymbol{e}_{3} \otimes \boldsymbol{e}_{3}\right) \cdot\left[\left(\mathcal{C}: \boldsymbol{\varepsilon}_{S}\left[\boldsymbol{u}_{1}\right]+\mathbf{d} \cdot \boldsymbol{\nabla}_{S} \varphi_{1}\right) \cdot \boldsymbol{n}\right] & \hat{\boldsymbol{x}} \in \Gamma_{0} \times(0, h), \quad t>0, \\
\quad=-\left(\boldsymbol{I}-\boldsymbol{e}_{3} \otimes \boldsymbol{e}_{3}\right) \cdot\left[\left(\mathcal{C}: \boldsymbol{\varepsilon}_{3}\left[\boldsymbol{u}_{0}\right]+\mathbf{d} \cdot \boldsymbol{e}_{3} \partial_{x_{3}} \varphi_{0}\right) \cdot \boldsymbol{n}\right] & \hat{\boldsymbol{x}} \in \Gamma_{0} \times(0, h), \quad t>0 .
\end{array}\right.
$$


One can check that the compatibility conditions of Proposition 3.2 are satisfied implying that, for each $t>0$ and $x_{3} \in(0, h)$, the function $\left(\varphi_{1}, \boldsymbol{u}_{1}\right)$ exists and is uniquely defined up to an additive constant denoted by $\phi_{1}\left(x_{3}, t\right)$ and an element $\boldsymbol{t}_{1}\left(x_{3}, t\right)+r_{1}\left(x_{3}, t\right) \boldsymbol{m}\left(\hat{x}_{1}, \hat{x}_{2}\right)$ of $\mathcal{E}_{S, 0}$ with the vector function $\boldsymbol{t}_{1}$ defined as

$$
\boldsymbol{t}_{1}\left(x_{3}, t\right)=\sum_{i=1}^{d} t_{1, i}\left(x_{3}, t\right) \boldsymbol{e}_{i} \text {. }
$$

Moreover as the right-hand side terms of (24) and (25) are constituted by the solution $\left(\varphi_{0}, \boldsymbol{u}_{0}\right)$ and on noting that we can decompose the term $\varepsilon_{3}\left[\boldsymbol{u}_{0}\right]$ as

$$
\varepsilon_{3}\left[\boldsymbol{u}_{0}(\hat{\boldsymbol{x}}, t)\right]=\sum_{i=1}^{d} \partial_{x_{3}}\left[t_{0, i}\left(x_{3}, t\right)\right] \varepsilon_{3}\left[x_{3} \boldsymbol{e}_{i}\right]+\partial_{x_{3}} r_{0}\left(x_{3}, t\right) \varepsilon_{3}\left[x_{3} \boldsymbol{m}\left(\hat{x}_{1}, \hat{x}_{2}\right)\right]
$$

we seek a decomposition of $\left(\boldsymbol{u}_{1}, \varphi_{1}\right)$ under the form

$$
\left\{\begin{aligned}
& \varphi_{1}(\hat{\boldsymbol{x}}, t) \equiv \phi_{1}\left(x_{3}, t\right)+\partial_{x_{3}} \varphi_{0}\left(x_{3}, t\right) \Phi_{0}\left(\hat{x}_{1}, \hat{x}_{2}\right)+\sum_{i=1}^{d} \partial_{x_{3}}\left[t_{0, i}\left(x_{3}, t\right)\right] \Phi_{i}\left(\hat{x}_{1}, \hat{x}_{2}\right)+\partial_{x_{3}} r_{0}\left(x_{3}, t\right) \Phi_{4}\left(\hat{x}_{1}, \hat{x}_{2}\right) \\
& \boldsymbol{u}_{1}(\hat{\boldsymbol{x}}, t) \equiv \boldsymbol{t}_{1}\left(x_{3}, t\right)+\boldsymbol{m}\left(\hat{x}_{1}, \hat{x}_{2}\right) r_{1}\left(x_{3}, t\right)+\partial_{x_{3}} \varphi_{0}\left(x_{3}, t\right) \mathcal{V}_{0}\left(\hat{x}_{1}, \hat{x}_{2}\right) \\
&+\sum_{i=1}^{d} \partial_{x_{3}}\left[t_{0, i}\left(x_{3}, t\right)\right] \mathcal{V}_{i}\left(\hat{x}_{1}, \hat{x}_{2}\right)+\partial_{x_{3}} r_{0}\left(x_{3}, t\right) \mathcal{V}_{4}\left(\hat{x}_{1}, \hat{x}_{2}\right)
\end{aligned}\right.
$$

in terms of a set of canonical functions $\left\{\left(\Phi_{i}\left(\hat{x}_{1}, \hat{x}_{2}\right), \mathcal{V}_{i}\left(\hat{x}_{1}, \hat{x}_{2}\right)\right)\right\}$ which are defined hereafter. Remark that, as soon as $\left|\Gamma_{0}\right| \neq 0$, then $d=2$ and the canonical functions $\left(\Phi_{3}, \mathcal{V}_{3}\right)$ do not appear in (26), however they can still be defined (as shown in what follows).

Canonical functions on cross-section. We introduce the canonical functions $\left\{\left(\Phi_{i}, \mathcal{V}_{i}\right), i \in\{0, \ldots, 4\}\right\}$ as the solutions of

$$
\begin{cases}\operatorname{div}_{S}\left(\boldsymbol{\epsilon} \cdot \nabla_{S} \Phi_{i}-\mathbf{d}^{\top}: \boldsymbol{\varepsilon}_{S}\left[\mathcal{V}_{i}\right]\right)=-\operatorname{div}_{S} \boldsymbol{p}_{i}, & \hat{\boldsymbol{x}} \in S, \\ \operatorname{div}_{S}\left(\mathcal{C}: \boldsymbol{\varepsilon}_{S}\left[\mathcal{V}_{i}\right]+\mathbf{d} \cdot \nabla_{S} \Phi_{i}\right)=-\operatorname{div}_{S} \boldsymbol{q}_{i}, & \hat{\boldsymbol{x}} \in S,\end{cases}
$$

with

$$
\int_{S} \Phi_{i} \mathrm{~d} \hat{s}=0, \quad \int_{S} \mathcal{V}_{i} \cdot \boldsymbol{w} \mathrm{d} \hat{s}=0, \quad \text { for all } \boldsymbol{w} \in \mathcal{E}_{S, 0},
$$

and satisfying the boundary conditions

$$
\begin{cases}\left(\boldsymbol{\epsilon} \cdot \nabla_{S} \Phi_{i}-\mathbf{d}^{\top}: \boldsymbol{\varepsilon}_{S}\left[\mathcal{V}_{i}\right]\right) \cdot \boldsymbol{n}=-\boldsymbol{p}_{i} \cdot \boldsymbol{n}, & \hat{\boldsymbol{x}} \in \partial S, \\ \left(\mathcal{C}: \varepsilon_{S}\left[\mathcal{V}_{i}\right]+\mathbf{d} \cdot \nabla_{S} \Phi_{i}\right) \cdot \boldsymbol{n}=-\boldsymbol{q}_{i} \cdot \boldsymbol{n} & \hat{\boldsymbol{x}} \in \Gamma_{N}, \\ \left(\boldsymbol{I}-\boldsymbol{e}_{3} \otimes \boldsymbol{e}_{3}\right) \cdot\left[\left(\mathcal{C}: \varepsilon_{S}\left[\mathcal{V}_{i}\right]+\mathbf{d} \cdot \nabla_{S} \Phi_{i}\right) \cdot \boldsymbol{n}\right]=-\left(\boldsymbol{I}-\boldsymbol{e}_{3} \otimes \boldsymbol{e}_{3}\right) \cdot\left[\boldsymbol{q}_{i} \cdot \boldsymbol{n}\right] & \hat{\boldsymbol{x}} \in \Gamma_{0}, \\ \mathcal{V}_{i} \cdot \boldsymbol{e}_{3}=0 & \hat{\boldsymbol{x}} \in \Gamma_{0} .\end{cases}
$$

In the previous equations, the source terms $\left\{\boldsymbol{p}_{i}, \boldsymbol{q}_{i}\right\}$ are defined, for $i \in\{1,2,3\}$ by

$$
\begin{array}{ll}
\boldsymbol{p}_{0}=\boldsymbol{\epsilon} \cdot \boldsymbol{e}_{3}, & \boldsymbol{q}_{0}=\mathbf{d} \cdot \boldsymbol{e}_{3} \\
\boldsymbol{p}_{i}=-\mathbf{d}^{\top}: \boldsymbol{\varepsilon}_{3}\left[x_{3} \boldsymbol{e}_{i}\right], & \boldsymbol{q}_{i}=\mathcal{C}: \boldsymbol{\varepsilon}_{3}\left[x_{3} \boldsymbol{e}_{i}\right] \\
\boldsymbol{p}_{4}=-\mathbf{d}^{\top}: \boldsymbol{\varepsilon}_{3}\left[x_{3} \boldsymbol{m}\left(\hat{x}_{1}, \hat{x}_{2}\right)\right], & \boldsymbol{q}_{4}=\mathcal{C}: \boldsymbol{\varepsilon}_{3}\left[x_{3} \boldsymbol{m}\left(\hat{x}_{1}, \hat{x}_{2}\right)\right]
\end{array}
$$


By direct application of Proposition 3.2 we can show these functions exist and are uniquely determined. Moreover, the following lemma provides, in different cases, explicit expressions of $\mathcal{V}_{1}$ and $\mathcal{V}_{2}$ together with related useful relation:

Lemma 3.1 For $i \in\{1,2\}$, if either $\left|\Gamma_{0}\right|=0$ or $\hat{\boldsymbol{x}} \cdot \boldsymbol{e}_{i}$ is constant along $\Gamma_{0}$, then

$o$ there exists a constant $c_{i}$ such that $\mathcal{V}_{i}\left(\hat{x}_{1}, \hat{x}_{2}\right)=\left(c_{i}-\hat{\boldsymbol{x}} \cdot \boldsymbol{e}_{i}\right) \boldsymbol{e}_{3}$ and $\Phi_{i}=0$

o moreover we have $\varepsilon_{S}\left[\mathcal{V}_{i}\right]=-\varepsilon_{3}\left[x_{3} \boldsymbol{e}_{i}\right]$ in $S$.

Otherwise, one has $\varepsilon_{S}\left[\mathcal{V}_{i}\right] \neq-\varepsilon_{3}\left[x_{3} \boldsymbol{e}_{i}\right]$ in $S$.

Proof If the assumptions of the lemma are satisfied then one can check that $\mathcal{V}_{i}\left(\hat{x}_{1}, \hat{x}_{2}\right)=\left(c_{i}-\hat{\boldsymbol{x}} \cdot \boldsymbol{e}_{i}\right) \boldsymbol{e}_{3}$ is indeed solution of the canonical problem $\left(27,[29)\right.$ : To do so one can use the equality $\boldsymbol{\varepsilon}_{S}\left[\mathcal{V}_{i}\right]=-\boldsymbol{\varepsilon}_{3}\left[x_{3} \boldsymbol{e}_{i}\right]$, which is true taking into account the form of $\mathcal{V}_{i}$. Note that, if $\left|\Gamma_{0}\right|=0$, then from (28), the constants $c_{i}$ are chosen to guaranty the condition

$$
\int_{S} \mathcal{V}_{i} \cdot e_{3} \mathrm{~d} \hat{s}=0
$$

whereas if $\hat{\boldsymbol{x}} \cdot \boldsymbol{e}_{i}$ is constant along $\Gamma_{0}$, then the constants $c_{i}$ are chosen so as to satisfy $\mathcal{V}_{i} \cdot \boldsymbol{e}_{3}=0$ on $\Gamma_{0}$. The last statement of the lemma is shown by contradiction. Assuming $\boldsymbol{\varepsilon}_{S}\left[\boldsymbol{V}_{i}\right]=-\varepsilon_{3}\left[x_{3} \boldsymbol{e}_{i}\right]$ implies $\partial_{\hat{x}_{i}} \mathcal{V}_{i} \cdot \boldsymbol{e}_{3}=-1$ in $S$, which is not compatible with the boundary condition $\mathcal{V}_{i} \cdot \boldsymbol{e}_{3}=0$ on $\Gamma_{0}$ if $\hat{\boldsymbol{x}} \cdot \boldsymbol{e}_{i}$ is not constant along $\Gamma_{0}$, and of course if $\left|\Gamma_{0}\right| \neq 0$.

Reciprocity formulae. It will be useful for the ensuing analysis to derive energy-like identities associated to the problem 27,29 . First, by multiplying each of the equations 27 by the fields $\psi$ and $\boldsymbol{v}$ respectively, integrating over $S$ and using the boundary conditions, then one has for all $i \in\{0, \ldots, 4\}$,

$$
\left\{\begin{array}{l}
\int_{S}\left(\boldsymbol{\epsilon} \cdot \nabla_{S} \Phi_{i}-\mathbf{d}^{\top}: \varepsilon_{S}\left[\mathcal{V}_{i}\right]\right) \cdot \nabla_{S} \psi \mathrm{d} \hat{s}=-\int_{S} \boldsymbol{p}_{i} \cdot \nabla_{S} \psi \mathrm{d} \hat{s} \\
\int_{S}\left(\mathcal{C}: \varepsilon_{S}\left[\mathcal{V}_{i}\right]+\mathbf{d} \cdot \nabla_{S} \Phi_{i}\right): \varepsilon_{S}[\boldsymbol{v}] \mathrm{d} \hat{s}=-\int_{S} \boldsymbol{q}_{i}: \boldsymbol{\varepsilon}_{S}[\boldsymbol{v}] \mathrm{d} \hat{s}
\end{array}\right.
$$

Upon choosing $(\psi, \boldsymbol{v})=\left(\Phi_{j}, \mathcal{V}_{j}\right)$ in the above relations, then by summation of the two previous equations one can derive the following equation for all $(i, j) \in\{0, \ldots, 4\}^{2}$

$$
\begin{array}{r}
\int_{S}\left(\varepsilon_{S}\left[\mathcal{V}_{i}\right]: \mathcal{C}: \varepsilon_{S}\left[\mathcal{V}_{j}\right]+\nabla_{S} \Phi_{i} \cdot \boldsymbol{\epsilon} \cdot \nabla_{S} \Phi_{j}\right) \mathrm{d} \hat{s}+\int_{S}\left(\mathbf{d}^{\top}: \varepsilon_{S}\left[\mathcal{V}_{j}\right] \cdot \nabla_{S} \Phi_{i}-\mathbf{d}^{\top}: \varepsilon_{S}\left[\mathcal{V}_{i}\right] \cdot \nabla_{S} \Phi_{j}\right) \mathrm{d} \hat{s} \\
=-\int_{S}\left(\boldsymbol{p}_{i} \cdot \nabla_{S} \Phi_{j}+\boldsymbol{q}_{i}: \varepsilon_{S}\left[\mathcal{V}_{j}\right]\right) \mathrm{d} \hat{s}
\end{array}
$$

which by inverting the role of the indices $i$ and $j$ leads to the reciprocity formula

$$
\int_{S}\left(\varepsilon_{S}\left[\mathcal{V}_{i}\right]: \mathcal{C}: \varepsilon_{S}\left[\mathcal{V}_{j}\right]+\nabla_{S} \Phi_{i} \cdot \boldsymbol{\epsilon} \cdot \nabla_{S} \Phi_{j}\right) \mathrm{d} \hat{s}=-\frac{1}{2} \int_{S}\left(\boldsymbol{p}_{i} \cdot \nabla_{S} \Phi_{j}+\boldsymbol{p}_{j} \cdot \nabla_{S} \Phi_{i}+\boldsymbol{q}_{i}: \varepsilon_{S}\left[\mathcal{V}_{j}\right]+\boldsymbol{q}_{j}: \varepsilon_{S}\left[\mathcal{V}_{i}\right]\right) \mathrm{d} \hat{s}
$$


In a similar fashion, on choosing $(\psi, \boldsymbol{v})=\left(\Phi_{j}, \mathcal{V}_{j}\right)$ and substracting the two equations of (31), the following relation is obtained for all $(i, j) \in\{0, \ldots, 4\}^{2}$

$$
\begin{array}{r}
\int_{S}\left(\varepsilon_{S}\left[\mathcal{V}_{i}\right]: \mathcal{C}: \varepsilon_{S}\left[\mathcal{V}_{j}\right]-\nabla_{S} \Phi_{i} \cdot \boldsymbol{\epsilon} \cdot \nabla_{S} \Phi_{j}\right) \mathrm{d} \hat{s}+\int_{S}\left(\mathbf{d}^{\top}: \varepsilon_{S}\left[\mathcal{V}_{j}\right] \cdot \nabla_{S} \Phi_{i}+\mathbf{d}^{\top}: \varepsilon_{S}\left[\mathcal{V}_{i}\right] \cdot \nabla_{S} \Phi_{j}\right) \mathrm{d} \hat{s} \\
=\int_{S}\left(\boldsymbol{p}_{i} \cdot \boldsymbol{\nabla}_{S} \Phi_{j}-\boldsymbol{q}_{i}: \varepsilon_{S}\left[\mathcal{V}_{j}\right]\right) \mathrm{d} \hat{s},
\end{array}
$$

which finally implies

$$
\int_{S}\left(\boldsymbol{p}_{i} \cdot \nabla_{S} \Phi_{j}-\boldsymbol{q}_{i}: \varepsilon_{S}\left[\mathcal{V}_{j}\right]\right) \mathrm{d} \hat{s}=\int_{S}\left(\boldsymbol{p}_{j} \cdot \nabla_{S} \Phi_{i}-\boldsymbol{q}_{j}: \varepsilon_{S}\left[\mathcal{V}_{i}\right]\right) \mathrm{d} \hat{s}
$$

\section{Limit equations and homogenized material parameters}

\subsection{Derivation of limit problems}

By identifying the $\mathcal{O}\left(\delta^{2}\right)$ terms in $(16)$, we obtain for $\hat{\boldsymbol{x}} \in \Omega$ and $t>0$

$$
\left\{\begin{array}{c}
\operatorname{div}_{S}\left(\boldsymbol{\epsilon} \cdot \nabla_{S} \varphi_{2}-\mathbf{d}^{\top}: \varepsilon_{S}\left[\boldsymbol{u}_{2}\right]\right)+\operatorname{div}_{S}\left(\boldsymbol{\epsilon} \cdot \boldsymbol{e}_{3} \partial_{x_{3}} \varphi_{1}-\mathbf{d}^{\top}: \varepsilon_{3}\left[\boldsymbol{u}_{1}\right]\right) \\
+\operatorname{div}_{3}\left(\boldsymbol{\epsilon} \cdot \nabla_{S} \varphi_{1}-\mathbf{d}^{\top}: \varepsilon_{S}\left[\boldsymbol{u}_{1}\right]\right)+\operatorname{div}_{3}\left(\boldsymbol{\epsilon} \cdot \boldsymbol{e}_{3} \partial_{x_{3}} \varphi_{0}-\mathbf{d}^{\top}: \varepsilon_{3}\left[\boldsymbol{u}_{0}\right]\right)=0 \\
\rho \partial_{t}^{2} \boldsymbol{u}_{0}-\operatorname{div}_{S}\left(\mathcal{C}: \varepsilon_{S}\left[\boldsymbol{u}_{2}\right]+\mathbf{d} \cdot \nabla_{S} \varphi_{2}\right)-\operatorname{div}_{S}\left(\mathcal{C}: \varepsilon_{3}\left[\boldsymbol{u}_{1}\right]+\mathbf{d} \cdot \boldsymbol{e}_{3} \partial_{x_{3}} \varphi_{1}\right) \\
\quad-\operatorname{div}_{3}\left(\mathcal{C}: \varepsilon_{S}\left[\boldsymbol{u}_{1}\right]+\mathbf{d} \cdot \nabla_{S} \varphi_{1}\right)-\operatorname{div}_{3}\left(\mathcal{C}: \varepsilon_{3}\left[\boldsymbol{u}_{0}\right]+\mathbf{d} \cdot \boldsymbol{e}_{3} \partial_{x_{3}} \varphi_{0}\right)=\mathbf{0}
\end{array}\right.
$$

whereas from (17) we obtain the following boundary conditions on $\partial S \times(0, h)$

$$
\left\{\begin{array}{lll}
\left(\boldsymbol{\epsilon} \cdot \nabla_{S} \varphi_{2}-\mathbf{d}^{\top}: \boldsymbol{\varepsilon}_{S}\left[\boldsymbol{u}_{2}\right]\right) \cdot \boldsymbol{n}=-\left(\boldsymbol{\epsilon} \cdot \boldsymbol{e}_{3} \partial_{x_{3}} \varphi_{1}-\mathbf{d}^{\top}: \boldsymbol{\varepsilon}_{3}\left[\boldsymbol{u}_{1}\right]\right) \cdot \boldsymbol{n}, & \hat{\boldsymbol{x}} \in \partial S \times(0, h), & t>0, \\
\left(\mathcal{C}: \boldsymbol{\varepsilon}_{S}\left[\boldsymbol{u}_{2}\right]+\mathbf{d} \cdot \boldsymbol{\nabla}_{S} \varphi_{2}\right) \cdot \boldsymbol{n}=-\left(\mathcal{C}: \boldsymbol{\varepsilon}_{3}\left[\boldsymbol{u}_{1}\right]+\mathbf{d} \cdot \boldsymbol{e}_{3} \partial_{x_{3}} \varphi_{1}\right) \cdot \boldsymbol{n} & \hat{\boldsymbol{x}} \in \partial S \times(0, h), \quad t>0 .
\end{array}\right.
$$

Making explicit the compatibility conditions for 35,36 that are given in 22 , and introducing the vector

$$
\boldsymbol{T}_{0}:=\left[t_{0,1} \ldots t_{0, d}\right]^{\top}
$$

then we can derive a system of $d+2$ unidimensional wave-like equations for $\left(\phi_{0}, \boldsymbol{T}_{0}, r_{0}\right)$ of the form

$$
\begin{cases}\bar{\epsilon} \partial_{x_{3}}^{2} \phi_{0}-\overline{\mathbf{d}}_{\phi \boldsymbol{t}}^{\top} \cdot \partial_{x_{3}}^{2} \boldsymbol{T}_{0}-\overline{\mathrm{d}}_{\phi r} \partial_{x_{3}}^{2} r_{0}=0, & x_{3} \in(0, h), \quad t>0 \\ \bar{\rho}_{\boldsymbol{t}} \partial_{t}^{2} \boldsymbol{T}_{0}-\overline{\mathbf{C}}_{\boldsymbol{t} \boldsymbol{t}} \cdot \partial_{x_{3}}^{2} \boldsymbol{T}_{0}-\overline{\mathbf{c}}_{\boldsymbol{t} r} \partial_{x_{3}}^{2} r_{0}-\overline{\mathbf{d}}_{\boldsymbol{t} \phi} \partial_{x_{3}}^{2} \phi_{0}=\mathbf{0}, & x_{3} \in(0, h), \quad t>0 \\ \bar{\rho}_{r} \partial_{t}^{2} r_{0}-\overline{\mathbf{c}}_{r \boldsymbol{t}}^{\top} \cdot \partial_{x_{3}}^{2} \boldsymbol{T}_{0}-\overline{\mathrm{c}}_{r r} \partial_{x_{3}}^{2} r_{0}-\overline{\mathrm{d}}_{r \phi} \partial_{x_{3}}^{2} \phi_{0}=0, & x_{3} \in(0, h), \quad t>0\end{cases}
$$

with

$$
\overline{\mathbf{C}}_{\boldsymbol{t} \boldsymbol{t}} \in \mathbb{R}^{d \times d}, \quad \overline{\mathbf{c}}_{\boldsymbol{t} r}, \overline{\mathbf{c}}_{r \boldsymbol{t}}, \overline{\mathbf{d}}_{\boldsymbol{t} \phi}, \overline{\mathbf{d}}_{\phi \boldsymbol{t}} \in \mathbb{R}^{d}, \quad \bar{\rho}_{\boldsymbol{t}}, \bar{\rho}_{r}, \overline{\mathrm{c}}_{r r}, \overline{\mathrm{d}}_{r \phi}, \overline{\mathrm{d}}_{\phi r}, \bar{\epsilon} \in \mathbb{R} .
$$

which represent homogenized material parameters and therefore do not depend on $\hat{\boldsymbol{x}}$ anymore.

More precisely, the equations (37)-(i) and (37)-(ii) are obtained by integrating over $S$ the equations (35)(i) and (35)-(ii) respectively and using the boundary conditions (36) and the expansion (26). Similarly, the equation (37)-(iii) is obtained by multiplying the equation (35)-(ii) by $\boldsymbol{m}$ and then integrating over $S$. 
Remark 4.1 The equations (37)-(ii) and (37)-(iii) do not involve any coupling term between $\boldsymbol{T}_{0}$ and $r_{0}$ with second order partial derivatives with respect to the time variable such as $\partial_{t}^{2} r_{0}$ and $\partial_{t}^{2} \boldsymbol{T}_{0}$. This is a consequence of the assumptions (5):

$$
\int_{S} \rho \boldsymbol{m} d \hat{s}=\mathbf{0}
$$

Property 4.1 By calculation one obtains

$$
\bar{\rho}_{\boldsymbol{t}}=\int_{S} \rho d \hat{s} \quad \text { and } \quad \bar{\rho}_{r}=\int_{S} \rho|\boldsymbol{m}|^{2} d \hat{s} .
$$

Along the same lines, we provide next the expressions of the other coefficients entering the equation (37) together with corresponding symmetry properties.

Property 4.2 The homogenized permittivity coefficient reads

$$
\bar{\epsilon}=\int_{S}\left(e_{3}+\nabla_{S} \Phi_{0}\right) \cdot \boldsymbol{\epsilon} \cdot\left(e_{3}+\nabla_{S} \Phi_{0}\right) d \hat{s}+\int_{S} \varepsilon_{S}\left[\mathcal{V}_{0}\right]: \mathcal{C}: \varepsilon_{S}\left[\mathcal{V}_{0}\right] d \hat{s}
$$

and we have $\bar{\epsilon} \geq S^{2} \epsilon_{-}>0$.

Proof By direct computation, one finds

$$
\bar{\epsilon}=\int_{S}\left\{\boldsymbol{e}_{3} \cdot \boldsymbol{\epsilon} \cdot \boldsymbol{e}_{3}+\boldsymbol{e}_{3} \cdot\left(\boldsymbol{\epsilon} \cdot \nabla_{S} \Phi_{0}-\mathbf{d}^{\top}: \varepsilon_{S}\left[\mathcal{V}_{0}\right]\right)\right\} \mathrm{d} \hat{s}
$$

Using equation $(32)$, with $i=j=0$ we can write

$$
-\int_{S} \boldsymbol{e}_{3} \cdot \mathbf{d}^{\top}: \boldsymbol{\varepsilon}_{S}\left[\mathcal{V}_{0}\right] \mathrm{d} \hat{s}=\int_{S} \boldsymbol{e}_{3} \cdot \boldsymbol{\epsilon} \cdot \boldsymbol{\nabla}_{S} \Phi_{0} \mathrm{~d} \hat{s}+\int_{S}\left(\varepsilon_{S}\left[\mathcal{V}_{0}\right]: \mathcal{C}: \boldsymbol{\varepsilon}_{S}\left[\mathcal{V}_{0}\right]+\nabla_{S} \Phi_{0} \cdot \boldsymbol{\epsilon} \cdot \nabla_{S} \Phi_{0}\right) \mathrm{d} \hat{s},
$$

which can be used to simplify the expression (39) to obtain (38). Finally, the estimate on $\bar{\epsilon}$ is obtained by dropping the positive contribution of the elastic energy and noticing, based on the structure of the operator $\nabla_{S}$, that

$$
\left\|e_{3}+\nabla_{S} \Phi_{0}\right\|_{L^{2}(S)} \geq\left\|e_{3}\right\|_{L^{2}(S)}
$$

Property 4.3 The homogenized elasticity coefficients are such that $\overline{\mathbf{C}}_{\boldsymbol{t} \boldsymbol{t}}=\overline{\mathbf{C}}_{\boldsymbol{t} \boldsymbol{t}}^{\top}$ with, for $(i, j) \in\{1, \ldots, d\}^{2}$

$$
\overline{\mathbf{C}}_{\boldsymbol{t} \boldsymbol{t}}:\left(\boldsymbol{e}_{i} \otimes \boldsymbol{e}_{j}\right)=\int_{S}\left(\varepsilon_{3}\left[x_{3} \boldsymbol{e}_{i}\right]+\boldsymbol{\varepsilon}_{S}\left[\mathcal{V}_{i}\right]\right): \mathcal{C}:\left(\varepsilon_{3}\left[x_{3} \boldsymbol{e}_{j}\right]+\varepsilon_{S}\left[\mathcal{V}_{j}\right]\right) d \hat{s}+\int_{S} \boldsymbol{\nabla}_{S} \Phi_{i} \cdot \boldsymbol{\epsilon} \cdot \nabla_{S} \Phi_{j} d \hat{s}
$$

as well as $\overline{\mathbf{c}}_{\boldsymbol{t} r}=\overline{\mathbf{c}}_{r \boldsymbol{t}}$ where

$$
\overline{\mathbf{c}}_{\boldsymbol{t} r} \cdot \boldsymbol{e}_{i}=\int_{S}\left(\varepsilon_{3}\left[x_{3} \boldsymbol{e}_{i}\right]+\varepsilon_{S}\left[\mathcal{V}_{i}\right]\right): \mathcal{C}:\left(\varepsilon_{3}\left[x_{3} \boldsymbol{m}\right]+\varepsilon_{S}\left[\mathcal{V}_{4}\right]\right) d \hat{s}+\int_{S} \nabla_{S} \Phi_{i} \cdot \boldsymbol{\epsilon} \cdot \nabla_{S} \Phi_{4} d \hat{s}
$$

and finally

$$
\overline{\mathrm{c}}_{r r}=\int_{S}\left(\varepsilon_{3}\left[x_{3} \boldsymbol{m}\right]+\varepsilon_{S}\left[\mathcal{V}_{4}\right]\right): \mathcal{C}:\left(\varepsilon_{3}\left[x_{3} \boldsymbol{m}\right]+\varepsilon_{S}\left[\mathcal{V}_{4}\right]\right) d \hat{s}+\int_{S} \nabla_{S} \Phi_{4} \cdot \boldsymbol{\epsilon} \cdot \nabla_{S} \Phi_{4} d \hat{s}
$$


Proof On noting that, for all symmetric second-order tensor $\boldsymbol{\sigma}$ one has

$$
\varepsilon_{3}\left[x_{3} \boldsymbol{e}_{i}\right]: \boldsymbol{\sigma}=\boldsymbol{e}_{i} \cdot \boldsymbol{\sigma} \cdot \boldsymbol{e}_{3} \quad \text { and } \quad \varepsilon_{3}\left[x_{3} \boldsymbol{m}\right]: \boldsymbol{\sigma}=\boldsymbol{m} \cdot \boldsymbol{\sigma} \cdot \boldsymbol{e}_{3},
$$

then by direct calculation, the elasticity coefficient $\overline{\mathbf{C}}_{\boldsymbol{t} \boldsymbol{t}}$ in 37 is found to be expressed as

$$
\overline{\mathbf{C}}_{\boldsymbol{t} \boldsymbol{t}}:\left(\boldsymbol{e}_{i} \otimes \boldsymbol{e}_{j}\right)=\int_{S}\left\{\boldsymbol{\varepsilon}_{3}\left[x_{3} \boldsymbol{e}_{i}\right]: \mathcal{C}: \varepsilon_{3}\left[x_{3} \boldsymbol{e}_{j}\right]+\varepsilon_{3}\left[x_{3} \boldsymbol{e}_{i}\right]:\left(\mathcal{C}: \boldsymbol{\varepsilon}_{S}\left[\mathcal{V}_{j}\right]+\mathbf{d} \cdot \nabla_{S} \Phi_{j}\right)\right\} \mathrm{d} \hat{s}
$$

Then, the symmetry of $\overline{\mathbf{C}}_{\boldsymbol{t} \boldsymbol{t}}$ is a direct consequence of the formula 34 which, for $(i, j) \in\{1, \ldots, d\}^{2}$, reduces to

$$
\int_{S} \varepsilon_{3}\left[x_{3} \boldsymbol{e}_{i}\right]:\left(\mathcal{C}: \varepsilon_{S}\left[\mathcal{V}_{j}\right]+\mathbf{d} \cdot \nabla_{S} \Phi_{j}\right) \mathrm{d} \hat{s}=\int_{S} \varepsilon_{3}\left[x_{3} \boldsymbol{e}_{j}\right]:\left(\mathcal{C}: \varepsilon_{S}\left[\mathcal{V}_{i}\right]+\mathbf{d} \cdot \nabla_{S} \Phi_{i}\right) \mathrm{d} \hat{s}
$$

Then, since $\overline{\mathbf{C}}_{\boldsymbol{t} \boldsymbol{t}}=\overline{\mathbf{C}}_{\boldsymbol{t} \boldsymbol{t}}^{\top}$, its component $(i, j)$ for all $(i, j) \in\{1, \ldots, d\}^{2}$ can be rewritten in symmetrized form as

$$
\begin{aligned}
\overline{\mathbf{C}}_{\boldsymbol{t} \boldsymbol{t}}:\left(\boldsymbol{e}_{i} \otimes \boldsymbol{e}_{j}\right)= & \int_{S} \varepsilon_{3}\left[x_{3} \boldsymbol{e}_{i}\right]: \mathcal{C}: \varepsilon_{3}\left[x_{3} \boldsymbol{e}_{j}\right] \mathrm{d} \hat{s} \\
& +\frac{1}{2} \int_{S}\left\{\varepsilon_{3}\left[x_{3} \boldsymbol{e}_{i}\right]:\left(\mathcal{C}: \varepsilon_{S}\left[\mathcal{V}_{j}\right]+\mathbf{d} \cdot \nabla_{S} \Phi_{j}\right)+\varepsilon_{3}\left[x_{3} \boldsymbol{e}_{j}\right]:\left(\mathcal{C}: \varepsilon_{S}\left[\mathcal{V}_{i}\right]+\mathbf{d} \cdot \nabla_{S} \Phi_{i}\right)\right\} \mathrm{d} \hat{s}
\end{aligned}
$$

and owing to the reciprocity formula 33 which implies

$$
\begin{array}{r}
\frac{1}{2} \int_{S}\left(\varepsilon_{3}\left[x_{3} \boldsymbol{e}_{i}\right]: \mathbf{d} \cdot \nabla_{S} \Phi_{j}+\varepsilon_{3}\left[x_{3} \boldsymbol{e}_{j}\right]: \mathbf{d} \cdot \nabla_{S} \Phi_{i}\right) \mathrm{d} \hat{s}=\frac{1}{2} \int_{S}\left(\varepsilon_{3}\left[x_{3} \boldsymbol{e}_{i}\right]: \mathcal{C}: \varepsilon_{S}\left[\mathcal{V}_{j}\right]+\varepsilon_{3}\left[x_{3} \boldsymbol{e}_{j}\right]: \mathcal{C}: \varepsilon_{S}\left[\mathcal{V}_{i}\right]\right) \mathrm{d} \hat{s} \\
+\int_{S}\left(\varepsilon_{S}\left[\mathcal{V}_{i}\right]: \mathcal{C}: \varepsilon_{S}\left[\mathcal{V}_{j}\right]+\nabla_{S} \Phi_{i} \cdot \boldsymbol{\epsilon} \cdot \nabla_{S} \Phi_{j}\right) \mathrm{d} \hat{s}
\end{array}
$$

the equation (43) can finally be recast in 40. Similarly, one finds for $i \in\{1, \ldots, d\}$

$$
\begin{aligned}
& \overline{\mathbf{c}}_{\boldsymbol{t} r} \cdot \boldsymbol{e}_{i}=\int_{S}\left\{\varepsilon_{3}\left[x_{3} \boldsymbol{e}_{i}\right]: \mathcal{C}: \varepsilon_{3}\left[x_{3} \boldsymbol{m}\right]+\varepsilon_{3}\left[x_{3} \boldsymbol{e}_{i}\right]:\left(\mathcal{C}: \boldsymbol{\varepsilon}_{S}\left[\mathcal{V}_{4}\right]+\mathbf{d} \cdot \nabla_{S} \Phi_{4}\right)\right\} \mathrm{d} \hat{s}, \\
& \overline{\mathbf{c}}_{r \boldsymbol{t}} \cdot \boldsymbol{e}_{i}=\int_{S}\left\{\varepsilon_{3}\left[x_{3} \boldsymbol{e}_{i}\right]: \mathcal{C}: \varepsilon_{3}\left[x_{3} \boldsymbol{m}\right]+\varepsilon_{3}\left[x_{3} \boldsymbol{m}\right]:\left(\mathcal{C}: \varepsilon_{S}\left[\mathcal{V}_{i}\right]+\mathbf{d} \cdot \nabla_{S} \Phi_{i}\right)\right\} \mathrm{d} \hat{s},
\end{aligned}
$$

and the equality $\overline{\mathbf{c}}_{\boldsymbol{t} r}=\overline{\mathbf{c}}_{r \boldsymbol{t}}$ is also a consequence of the formula 34 with $i=4$ and $j \in\{1, \ldots, d\}$. Using the same algebraic manipulations derived from (33), one can rewrite this parameter as in 41 . Finally, direct calculations lead to

$$
\overline{\mathrm{c}}_{r r}=\int_{S}\left\{\varepsilon_{3}\left[x_{3} \boldsymbol{m}\right]: \mathcal{C}: \varepsilon_{3}\left[x_{3} \boldsymbol{m}\right]+\varepsilon_{3}\left[x_{3} \boldsymbol{m}\right]:\left(\mathcal{C}: \boldsymbol{\varepsilon}_{S}\left[\mathcal{V}_{4}\right]+\mathbf{d} \cdot \nabla_{S} \Phi_{4}\right)\right\} \mathrm{d} \hat{s},
$$

and as in the above relations, we can obtain the alternative formula 42 .

Property 4.4 The homogenized piezoelectric terms are such that $\overline{\mathbf{d}}_{\phi \boldsymbol{t}}=\overline{\mathbf{d}}_{\boldsymbol{t} \phi} \equiv \overline{\mathbf{d}}_{\boldsymbol{t}}$ and $\overline{\mathrm{d}}_{\phi r}=\overline{\mathrm{d}}_{r \phi} \equiv \overline{\mathrm{d}}_{r}$ with

$$
\overline{\mathbf{d}}_{\boldsymbol{t}} \cdot \boldsymbol{e}_{i}=\int_{S}\left(\varepsilon_{3}\left[x_{3} \boldsymbol{e}_{i}\right]+\varepsilon_{S}\left[\mathcal{V}_{i}\right]\right):\left(\mathbf{d} \cdot \boldsymbol{e}_{3}+\mathcal{C}: \varepsilon_{S}\left[\mathcal{V}_{0}\right]\right) d \hat{s}+\int_{S} \nabla_{S} \Phi_{i} \cdot \boldsymbol{\epsilon} \cdot \nabla_{S} \Phi_{0} d \hat{s}
$$

and

$$
\overline{\mathrm{d}}_{r}=\int_{S}\left(\varepsilon_{3}\left[x_{3} \boldsymbol{m}\right]+\varepsilon_{S}\left[\mathcal{V}_{4}\right]\right):\left(\mathbf{d} \cdot \boldsymbol{e}_{3}+\mathcal{C}: \varepsilon_{S}\left[\mathcal{V}_{0}\right]\right) d \hat{s}+\int_{S} \nabla_{S} \Phi_{4} \cdot \boldsymbol{\epsilon} \cdot \nabla_{S} \Phi_{0} d \hat{s}
$$


Proof The coupling terms between $\boldsymbol{T}_{0}$ and $\phi_{0}$ are readily obtained as, for $i \in\{1, \ldots, d\}$

$$
\begin{aligned}
& \overline{\mathbf{d}}_{\phi \boldsymbol{t}} \cdot \boldsymbol{e}_{i}=\int_{S}\left\{\boldsymbol{e}_{3} \cdot \mathbf{d}^{\top}: \varepsilon_{3}\left[x_{3} \boldsymbol{e}_{i}\right]+\boldsymbol{e}_{3} \cdot\left(\mathbf{d}^{\top}: \boldsymbol{\varepsilon}_{S}\left[\mathcal{V}_{i}\right]-\boldsymbol{\epsilon} \cdot \boldsymbol{\nabla}_{S} \Phi_{i}\right)\right\} \mathrm{d} \hat{s}, \\
& \overline{\mathbf{d}}_{\boldsymbol{t} \phi} \cdot \boldsymbol{e}_{i}=\int_{S}\left\{\varepsilon_{3}\left[x_{3} \boldsymbol{e}_{i}\right]: \mathbf{d} \cdot \boldsymbol{e}_{3}+\varepsilon_{3}\left[x_{3} \boldsymbol{e}_{i}\right]:\left(\mathcal{C}: \varepsilon_{S}\left[\mathcal{V}_{0}\right]+\mathbf{d} \cdot \nabla_{S} \Phi_{0}\right)\right\} \mathrm{d} \hat{s} .
\end{aligned}
$$

Then, as in the above equations, using the relation (34) with $i=0$ and $j \in\{1, \ldots, d\}$ one can prove that $\overline{\mathbf{d}}_{\phi \boldsymbol{t}}=\overline{\mathbf{d}}_{\boldsymbol{t} \phi}$ and these terms can be recast in (44) using 33 . In the same way the piezoelectric terms that couple the terms $r_{0}$ and $\phi_{0}$ reads

$$
\begin{aligned}
& \overline{\mathrm{d}}_{\phi r}=\int_{S}\left\{\boldsymbol{e}_{3} \cdot \mathbf{d}^{\top}: \varepsilon_{3}\left[x_{3} \boldsymbol{m}\right]+\boldsymbol{e}_{3} \cdot\left(\mathbf{d}^{\top}: \boldsymbol{\varepsilon}_{S}\left[\mathcal{V}_{4}\right]-\boldsymbol{\epsilon} \cdot \nabla_{S} \Phi_{4}\right)\right\} \mathrm{d} \hat{s}, \\
& \overline{\mathrm{d}}_{r \phi}=\int_{S}\left\{\boldsymbol{\varepsilon}_{3}\left[x_{3} \boldsymbol{m}\right]: \mathbf{d} \cdot \boldsymbol{e}_{3}+\varepsilon_{3}\left[x_{3} \boldsymbol{m}\right]:\left(\mathcal{C}: \boldsymbol{\varepsilon}_{S}\left[\mathcal{V}_{0}\right]+\mathbf{d} \cdot \nabla_{S} \Phi_{0}\right)\right\} \mathrm{d} \hat{s} .
\end{aligned}
$$

This symmetry properties are once again direct consequence of the relation (34).

\subsection{Simplification of some homogenized parameters}

\subsubsection{Boundary conditions effects}

In this section we analyze the homogenized material parameters obtained previously depending on the choice of the boundary conditions.

Case $\left|\Gamma_{0}\right| \neq 0$ with $\hat{\boldsymbol{x}} \cdot \boldsymbol{e}_{i}$ non-constant on $\Gamma_{0}$ for $i=1$ and 2 . In this configuration where $d=2$ and which in particular includes the case $\Gamma_{0}=\partial S$, we can prove the following positivity property

Lemma 4.1 The $3 \times 3$ matrix $\left(\begin{array}{cc}\overline{\mathbf{C}}_{\boldsymbol{t} \boldsymbol{t}} & \overline{\mathbf{c}}_{\boldsymbol{t} r} \\ \overline{\mathbf{c}}_{\boldsymbol{t} r}^{\top} & \overline{\mathbf{c}}_{r r}\end{array}\right)$ is symmetric definite positive.

Proof Let us define

$$
\boldsymbol{u}=\sum_{i=1}^{2} t_{i} \boldsymbol{e}_{i}+r \boldsymbol{m}, \quad \mathcal{V}=\sum_{i=1}^{2} t_{i} \mathcal{V}_{i}+r \mathcal{V}_{4} \quad \text { and } \quad \Phi=\sum_{i=1}^{2} t_{i} \Phi_{i}+r \Phi_{4}
$$

where $t_{1}, t_{2}$ and $r$ are positive scalars. Using the equations 40 and (41) we find

$$
\begin{aligned}
\left(\begin{array}{lll}
t_{1} & t_{2} & r
\end{array}\right) \cdot\left(\begin{array}{cc}
\overline{\mathbf{C}}_{\boldsymbol{t t}} & \overline{\mathbf{c}}_{\boldsymbol{t} r} \\
\overline{\mathbf{c}}_{\boldsymbol{t} r}^{\top} & \overline{\mathrm{c}}_{r r}
\end{array}\right) \cdot\left(\begin{array}{c}
t_{1} \\
t_{2} \\
r
\end{array}\right) & =\int_{S}\left(\varepsilon_{3}\left[x_{3} \boldsymbol{u}\right]+\boldsymbol{\varepsilon}_{S}[\mathcal{V}]\right): \mathcal{C}:\left(\varepsilon_{3}\left[x_{3} \boldsymbol{u}\right]+\varepsilon_{S}[\mathcal{V}]\right) \mathrm{d} \hat{s}+\int_{S} \nabla_{S} \Phi \cdot \boldsymbol{\epsilon} \cdot \nabla_{S} \Phi \mathrm{d} \hat{s} \\
& \geq C_{-}\left\|\varepsilon_{3}\left[x_{3} \boldsymbol{u}\right]+\varepsilon_{S}[\mathcal{V}]\right\|_{L^{2}(S)}^{2} .
\end{aligned}
$$

We now prove that $\left\|\varepsilon_{3}\left[x_{3} \boldsymbol{u}\right]+\varepsilon_{S}[\mathcal{V}]\right\|_{L^{2}(S)}^{2}>0$ for all $\left(t_{1}, t_{2}, r\right) \in \mathbb{R}^{3} \backslash\{(0,0,0)\}$ in order to finish the proof. Assume that there exist $\left(t_{1}, t_{2}, r\right) \neq(0,0,0)$ such that $\varepsilon_{3}\left[x_{3} \boldsymbol{u}\right]+\varepsilon_{S}[\mathcal{V}]=\mathbf{0}$, then by definition of the operators $\varepsilon_{3}$ and $\varepsilon_{S}$ we have

$$
\partial_{\hat{x}_{1}} \mathcal{V} \cdot \boldsymbol{e}_{3}=t_{1}+r \hat{x}_{2}, \quad \partial_{\hat{x}_{2}} \mathcal{V} \cdot \boldsymbol{e}_{3}=t_{2}-r \hat{x}_{1}
$$

which imply, after integration

$$
\mathcal{V} \cdot \boldsymbol{e}_{3}=t_{1} \hat{x}_{1}+t_{2} \hat{x}_{2}+r \hat{x}_{1} \hat{x}_{2}+c_{1} \text { and } \mathcal{V} \cdot \boldsymbol{e}_{3}=t_{1} \hat{x}_{1}+t_{2} \hat{x}_{2}-r \hat{x}_{1} \hat{x}_{2}+c_{2}
$$


where $c_{1}$ and $c_{2}$ ) are two constants. By identification, we necessarily have $r=0$. Moreover, as $\mathcal{V} \cdot \boldsymbol{e}_{3}$ must vanish along $\Gamma_{0}$ and since both $\hat{x}_{1}$ and $\hat{x}_{2}$ are non-constant along $\Gamma_{0}$ (in the opposite case, studied hereafter, we can tune $c_{1}$ or $c_{2}$ such that the boundary condition is satisfied) we also have $t_{1}=t_{2}=0$ which contradicts $\left(t_{1}, t_{2}, r\right) \neq(0,0,0)$.

Case $\left|\Gamma_{0}\right|=0$. In this situation there is no imposed mixed elastic boundary condition, i.e. the lateral surface $\partial S \times(0, h)$ is only associated with a traction-free boundary condition, then we show that the homogenized material parameters given previously can be simplified. The next lemma is an immediate consequence of Lemma 3.1 and of the results of the previous section.

Lemma 4.2 The homogenized elastic and piezoelectric coefficients are such that

$$
\overline{\mathbf{C}}_{\boldsymbol{t} \boldsymbol{t}}=\overline{\mathrm{c}}_{33} \boldsymbol{e}_{3} \otimes \boldsymbol{e}_{3}, \quad \overline{\mathbf{c}}_{\boldsymbol{t} r}=\overline{\mathbf{c}}_{r \boldsymbol{t}}=\overline{\mathrm{c}}_{\boldsymbol{t} r} \boldsymbol{e}_{3}, \quad \overline{\mathbf{d}}_{\phi \boldsymbol{t}}=\overline{\mathbf{d}}_{\boldsymbol{t} \phi}=\overline{\mathrm{d}}_{\boldsymbol{t}} \boldsymbol{e}_{3} .
$$

Moreover, the matrix $\left(\begin{array}{cc}\overline{\mathrm{c}}_{33} & \overline{\mathrm{c}}_{\boldsymbol{t} r} \\ \overline{\mathrm{c}}_{\boldsymbol{t} r} & \overline{\mathrm{c}}_{r r}\end{array}\right)$ is symmetric definite positive.

Proof Lemma 3.1 is valid in the case $\left|\Gamma_{0}\right|=0$, therefore we have $\boldsymbol{\varepsilon}_{S}\left[\mathcal{V}_{1}\right]=-\boldsymbol{\varepsilon}_{3}\left[x_{3} \boldsymbol{e}_{1}\right]$ and $\boldsymbol{\varepsilon}_{S}\left[\mathcal{V}_{2}\right]=-\boldsymbol{\varepsilon}_{3}\left[x_{3} \boldsymbol{e}_{2}\right]$, as well as $\Phi_{1}=\Phi_{2}=0$. Then the formula 40 for the homogenized coefficient show that all terms in $\overline{\mathbf{C}}_{\boldsymbol{t} t}$ turn out to be zero except $\overline{\mathbf{C}}_{\boldsymbol{t} \boldsymbol{t}}:\left(\boldsymbol{e}_{3} \otimes \boldsymbol{e}_{3}\right)$. The same arguments are used to show that $\overline{\mathbf{c}}_{\boldsymbol{t} r}=\overline{\mathrm{c}}_{\boldsymbol{t} r} \boldsymbol{e}_{3}$ from the equation (41) and $\overline{\mathbf{d}}_{\phi \boldsymbol{t}}=\overline{\mathbf{d}}_{\boldsymbol{t} \phi}=\overline{\mathrm{d}}_{\boldsymbol{t}} \boldsymbol{e}_{3}$ owing to the equation (44). The positivity property can be proven in a straightforward manner as in Lemma 4.1 .

Case $\left|\Gamma_{0}\right| \neq 0$ with $\hat{\boldsymbol{x}} \cdot \boldsymbol{e}_{i}$ constant on $\Gamma_{0}$ for $i=1$ or $i=2$. This hypothesis corresponds to an intermediate configuration between the two cases above. Consider, for example, the case $i=2$ while the case $\hat{x}_{1}$ constant on $\Gamma_{0}$ yields similar developments and is omitted for brevity. We have $d=2$, therefore the component along the $z$-axis of the displacement field vanishes. Moreover, application of Lemma 3.1 entails

$$
\varepsilon_{S}\left[\mathcal{V}_{1}\right] \neq-\varepsilon_{3}\left[x_{3} \boldsymbol{e}_{1}\right] \quad \text { while } \quad \varepsilon_{S}\left[\mathcal{V}_{2}\right]=-\varepsilon_{3}\left[x_{3} \boldsymbol{e}_{2}\right]
$$

Then, using the same approach as in the first case above, one can show that these relations lead to

Lemma 4.3 The homogenized elastic and piezoelectric coefficients are such that

$$
\overline{\mathbf{C}}_{\boldsymbol{t} \boldsymbol{t}}=\overline{\mathrm{c}}_{11} \boldsymbol{e}_{1} \otimes \boldsymbol{e}_{1}, \quad \overline{\mathbf{c}}_{\boldsymbol{t} r}=\overline{\mathbf{c}}_{r \boldsymbol{t}}=\overline{\mathrm{c}}_{\boldsymbol{t} r} \boldsymbol{e}_{1}, \quad \overline{\mathbf{d}}_{\phi \boldsymbol{t}}=\overline{\mathbf{d}}_{\boldsymbol{t} \phi}=\overline{\mathrm{d}}_{\boldsymbol{t}} \boldsymbol{e}_{1} .
$$

Moreover, the matrix $\left(\begin{array}{cc}\overline{\mathrm{c}}_{11} & \overline{\mathrm{c}}_{\boldsymbol{t} r} \\ \overline{\mathrm{c}}_{\boldsymbol{t} r} & \overline{\mathrm{c}}_{r r}\end{array}\right)$ is symmetric definite positive.

\subsubsection{Homogeneous case with free surface boundary condition}

In this section we discuss the simplification of some homogenized parameters that occur when assuming that the piezoelectric bar is homogeneous.

Theorem 4.1 If $\left|\Gamma_{0}\right|=0$ and all the material parameters $(\rho, \boldsymbol{\epsilon}, \mathcal{C}, \mathbf{d})$ are constant in $S$, then the canonical functions $\left(\Phi_{i}, \mathcal{V}_{i}\right)$ for $i \in\{0, \ldots, 3\}$ are linear in the coordinates $\hat{x}_{1}$ and $\hat{x}_{2}$. 
Proof When $i \in\{1,2\}$ this result is a direct consequence of Lemma 3.1. For the case $i \in\{0,3\}$ we provide hereafter a constructive proof. As a preliminary, let us define the spaces

$$
\mathcal{L}_{\text {sym }, 0}\left(\mathbb{R}^{3}\right):=\left\{\mathcal{E} \in \mathcal{L}_{\text {sym }}\left(\mathbb{R}^{3}\right), \quad \mathcal{E}: \boldsymbol{e}_{3} \otimes \boldsymbol{e}_{3}=0\right\} \quad \text { and } \quad \mathbb{R}_{0}^{3}:=\left\{\boldsymbol{\Psi} \in \mathbb{R}^{3}, \quad \boldsymbol{\Psi} \cdot \boldsymbol{e}_{3}=0\right\} .
$$

Omitting some details for brevity, we prove that for all $(\boldsymbol{p}, \boldsymbol{q}) \in \mathbb{R}^{3} \times \mathcal{L}_{\text {sym }}\left(\mathbb{R}^{3}\right)$, there exists a unique $(\boldsymbol{\Psi}, \mathcal{E}) \in$ $\mathbb{R}_{0}^{3} \times \mathcal{L}_{\mathrm{sym}, 0}\left(\mathbb{R}^{3}\right)$ such that for all $\boldsymbol{n} \in \mathbb{R}_{0}^{3}$

$$
\begin{aligned}
& \left(\boldsymbol{\epsilon} \cdot \boldsymbol{\Psi}-\mathrm{d}^{\top}: \mathcal{E}\right) \cdot \boldsymbol{n}=\boldsymbol{p} \cdot \boldsymbol{n} \\
& (\mathcal{C}: \mathcal{E}+\mathrm{d} \cdot \boldsymbol{\Psi}) \cdot \boldsymbol{n}=\boldsymbol{q} \cdot \boldsymbol{n} .
\end{aligned}
$$

First, one introduces the matrix

$$
\mathbf{Q}=\left(\begin{array}{ccccccc}
\epsilon_{11} & \epsilon_{12} & -\mathrm{d}_{11} & -\mathrm{d}_{12} & -\sqrt{2} \mathrm{~d}_{14} & -\sqrt{2} \mathrm{~d}_{15} & -\sqrt{2} \mathrm{~d}_{16} \\
\epsilon_{12} & \epsilon_{22} & -\mathrm{d}_{21} & -\mathrm{d}_{22} & -\sqrt{2} \mathrm{~d}_{24} & -\sqrt{2} \mathrm{~d}_{25} & -\sqrt{2} \mathrm{~d}_{26} \\
\mathrm{~d}_{11} & \mathrm{~d}_{21} & \mathcal{C}_{11} & \mathcal{C}_{12} & \sqrt{2} \mathcal{C}_{14} & \sqrt{2} \mathcal{C}_{15} & \sqrt{2} \mathcal{C}_{16} \\
\mathrm{~d}_{12} & \mathrm{~d}_{22} & \mathcal{C}_{12} & \mathcal{C}_{22} & \sqrt{2} \mathcal{C}_{24} & \sqrt{2} \mathcal{C}_{25} & \sqrt{2} \mathcal{C}_{26} \\
\sqrt{2} \mathrm{~d}_{14} & \sqrt{2} \mathrm{~d}_{24} & \sqrt{2} \mathcal{C}_{14} & \sqrt{2} \mathcal{C}_{24} & 2 \mathcal{C}_{44} & 2 \mathcal{C}_{45} & 2 \mathcal{C}_{46} \\
\sqrt{2} \mathrm{~d}_{15} & \sqrt{2} \mathrm{~d}_{25} & \sqrt{2} \mathcal{C}_{15} & \sqrt{2} \mathcal{C}_{25} & 2 \mathcal{C}_{45} & 2 \mathcal{C}_{55} & 2 \mathcal{C}_{56} \\
\sqrt{2} \mathrm{~d}_{16} & \sqrt{2} \mathrm{~d}_{26} & \sqrt{2} \mathcal{C}_{16} & \sqrt{2} \mathcal{C}_{26} & 2 \mathcal{C}_{46} & 2 \mathcal{C}_{56} & 2 \mathcal{C}_{66}
\end{array}\right)
$$

where we have used the Voigt notation for the tensors $\mathbf{d}$ and $\mathcal{C}$, i.e. taking into account usual symmetries, the in-parenthesis indices in $\mathrm{d}_{k(i j)}$ and $\mathcal{C}_{(i j)(k l)}$ are replaced according to the convention

$$
1 \leftrightarrow(11), \quad 2 \leftrightarrow(22), \quad 3 \leftrightarrow(33), \quad 4 \leftrightarrow(13) \text { or }(31), \quad 5 \leftrightarrow(23) \text { or }(32), \quad 6 \leftrightarrow(12) \text { or }(21)
$$

Next consider the vectors

$$
\mathbf{X}=\left(\begin{array}{c}
\boldsymbol{\Psi} \cdot \boldsymbol{e}_{1} \\
\boldsymbol{\Psi} \cdot \boldsymbol{e}_{2} \\
\mathcal{E}: \boldsymbol{e}_{1} \otimes \boldsymbol{e}_{1} \\
\mathcal{E}: \boldsymbol{e}_{2} \otimes \boldsymbol{e}_{2} \\
\sqrt{2} \mathcal{E}: \boldsymbol{e}_{2} \otimes \boldsymbol{e}_{3} \\
\sqrt{2} \mathcal{E}: \boldsymbol{e}_{1} \otimes \boldsymbol{e}_{3} \\
\sqrt{2} \mathcal{E}: \boldsymbol{e}_{1} \otimes \boldsymbol{e}_{2}
\end{array}\right) \quad \text { and } \quad \mathbf{Y}=\left(\begin{array}{c}
\boldsymbol{p} \cdot \boldsymbol{e}_{1} \\
\boldsymbol{p} \cdot \boldsymbol{e}_{2} \\
\boldsymbol{q}: \boldsymbol{e}_{1} \otimes \boldsymbol{e}_{1} \\
\boldsymbol{q}: \boldsymbol{e}_{2} \otimes \boldsymbol{e}_{2} \\
\sqrt{2} \boldsymbol{q}: \boldsymbol{e}_{2} \otimes \boldsymbol{e}_{3} \\
\sqrt{2} \boldsymbol{q}: \boldsymbol{e}_{1} \otimes \boldsymbol{e}_{3} \\
\sqrt{2} \boldsymbol{q}: \boldsymbol{e}_{1} \otimes \boldsymbol{e}_{2}
\end{array}\right)
$$

then $(\Psi, \mathcal{E})$ solution of 45 is defined through the inversion of the following linear system

$$
\mathrm{QX}=\mathbf{Y} .
$$

The existence of a unique $(\boldsymbol{\Psi}, \mathcal{E})$ solution of the previous equation is a direct consequence of the strict positivity properties of $\boldsymbol{\epsilon}$ and $\mathcal{C}$ as well as the consistency of quadratics forms written in tensor and matrix forms, i.e. we have

$$
\mathbf{X}^{\top} \mathbf{Q X}=\boldsymbol{\Psi} \cdot \boldsymbol{\epsilon} \cdot \boldsymbol{\Psi}+\mathcal{E}: \mathcal{C}: \mathcal{E} \geq \epsilon_{-}|\boldsymbol{\Psi}|^{2}+c_{-}|\mathcal{E}|^{2}>0, \quad \text { for all }(\boldsymbol{\Psi}, \mathcal{E}) \in \mathbb{R}_{0}^{3} \times \mathcal{L}_{\mathrm{sym}, 0}\left(\mathbb{R}^{3}\right) .
$$

As a second and final step we show that we can construct linear functions $(\Phi, \mathcal{V})$, with respect to $\hat{x}_{1}$ and $\hat{x}_{2}$, satisfying the compatibility conditions 28 and such that, for all $(\boldsymbol{\Psi}, \mathcal{E}) \in \mathbb{R}_{0}^{3} \times \mathcal{L}_{\text {sym, } 0}\left(\mathbb{R}^{3}\right)$ we have

$$
\nabla_{S} \Phi=\Psi \quad \text { and } \quad \varepsilon_{S}[\mathcal{V}]=\mathcal{E}
$$

Let us first remark that, as $\rho$ is constant, from (5) we have

$$
\int_{S} \hat{x}_{1} \mathrm{~d} \boldsymbol{x}=0 \quad \text { and } \quad \int_{S} \hat{x}_{2} \mathrm{~d} \boldsymbol{x}=0 .
$$


Obviously, one can find a linear function $\Phi$ such that the first equation of 48 is satisfied for any $\boldsymbol{\Psi} \in \mathbb{R}_{0}^{3}$. Moreover, the corresponding existence result for $\mathcal{V}$ is the consequence of the following properties. Let the space $\mathcal{W}$ of linear functions be defined by

$$
\mathcal{W}:=\operatorname{span}\left\{\left(\begin{array}{c}
\hat{x}_{1} \\
\hat{x}_{2} \\
0
\end{array}\right),\left(\begin{array}{c}
\hat{x}_{2} \\
c_{1} \hat{x}_{1} \\
0
\end{array}\right),\left(\begin{array}{c}
\hat{x}_{1} \\
c_{2} \hat{x}_{1} \\
0
\end{array}\right),\left(\begin{array}{c}
0 \\
0 \\
\hat{x}_{1}
\end{array}\right),\left(\begin{array}{c}
0 \\
0 \\
\hat{x}_{2}
\end{array}\right)\right\}
$$

with the constant parameters $c_{1}$ and $c_{2}$ given by

$$
c_{1}=\left(\int_{S} \hat{x}_{1}^{2} \mathrm{~d} \hat{\boldsymbol{x}}\right)^{-1} \int_{S} \hat{x}_{2}^{2} \mathrm{~d} \hat{\boldsymbol{x}} \quad \text { and } \quad c_{2}=\left(\int_{S} \hat{x}_{1}^{2} \mathrm{~d} \hat{\boldsymbol{x}}\right)^{-1} \int_{S} \hat{x}_{2} \hat{x}_{1} \mathrm{~d} \hat{\boldsymbol{x}} .
$$

Then, $\mathcal{W}$ is orthogonal to $\mathcal{E}_{S}$, in the sense of the $L^{2}$ scalar product on $S$, therefore the compatibility conditions (28) are satisfied. Moreover, on noting that one has formally $\varepsilon_{S}[\mathcal{W}] \equiv \mathcal{L}_{\text {sym }, 0}\left(\mathbb{R}^{3}\right)$, then for any $\mathcal{E} \in \mathcal{L}_{\text {sym }, 0}\left(\mathbb{R}^{3}\right)$ one can find $\mathcal{V} \in \mathcal{W}$ such that $\varepsilon_{S}[\mathcal{V}]=\mathcal{E}$.

Finally, the existence of the function $\left(\Phi_{i}, \mathcal{V}_{i}\right)$ can be deduced from a construction procedure based on the above derivations and one concludes owing to the uniqueness of the solution to the problem (27, 29): For each $i \in\{0,3\}$ the corresponding right hand side $\mathbf{Y}$ of 47$)$ is constructed using the constant function $\left(\boldsymbol{p}_{i}, \boldsymbol{q}_{i}\right)$ as defined by (30), then the vector $\mathbf{X}$ is computed by inversion of the linear system 46 . With $\mathbf{X}$ at hand, the functions $\mathcal{V}_{i}$ and $\Phi_{i}$ are finally recovered as linear functions of $\hat{x}_{1}$ and $\hat{x}_{2}$.

The previous theorem implies the following additional simplifications of the homogenized parameters

Corollary 4.1 If $\left|\Gamma_{0}\right|=0$ and all the physical coefficients $(\rho, \boldsymbol{\epsilon}, \mathcal{C}, \mathbf{d})$ are constant in $S$ then

$$
\overline{\mathbf{c}}_{\boldsymbol{t r}}=\overline{\mathbf{c}}_{r \boldsymbol{t}}=\mathbf{0}, \quad \overline{\mathrm{d}}_{\phi r}=\overline{\mathrm{d}}_{r \phi}=0
$$

Proof In the case where $\left|\Gamma_{0}\right|=0$, we already know from Lemma 4.2 that $\overline{\mathbf{c}}_{r \boldsymbol{t}} \cdot \boldsymbol{e}_{1}=\overline{\mathbf{c}}_{r \boldsymbol{t}} \cdot \boldsymbol{e}_{2}=0$. Moreover, we have

$$
\overline{\mathbf{c}}_{r \boldsymbol{t}} \cdot \boldsymbol{e}_{3}=\int_{S}\left\{\varepsilon_{3}\left[x_{3} \boldsymbol{e}_{3}\right]: \mathcal{C}: \varepsilon_{3}\left[x_{3} \boldsymbol{m}\right]+\varepsilon_{3}\left[x_{3} \boldsymbol{m}\right]:\left(\mathcal{C}: \varepsilon_{S}\left[\mathcal{V}_{3}\right]+\mathbf{d} \cdot \nabla_{S} \Phi_{3}\right)\right\} \mathrm{d} \hat{s}
$$

The theorem 4.1 shows that $\nabla_{S} \Phi_{3}$ and $\varepsilon_{S}\left[\mathcal{V}_{3}\right]$ are constant, therefore, upon rewriting the previous identity as

$$
\overline{\mathbf{c}}_{r \boldsymbol{t}} \cdot \boldsymbol{e}_{3}=\varepsilon_{3}\left[x_{3} \boldsymbol{e}_{3}\right]: \mathcal{C}: \varepsilon_{3}\left[x_{3} \int_{S} \boldsymbol{m} \mathrm{d} \hat{s}\right]+\varepsilon_{3}\left[x_{3} \int_{S} \boldsymbol{m} \mathrm{d} \hat{s}\right]:\left(\mathcal{C}: \varepsilon_{S}\left[\mathcal{V}_{3}\right]+\mathbf{d} \cdot \nabla_{S} \Phi_{3}\right),
$$

one obtains the sought results thanks to 49 . The same arguments also apply for the parameter $\overline{\mathrm{d}}_{\phi r}$.

\subsection{Complete 1D models involving boundary conditions}

To derive a complete $1 \mathrm{D}$ piezoelectric sensor model, one has to take into account the conditions imposed on the remaining part of the boundary, i.e. the elastic and electrical conditions at $x_{3}=h$ and $x_{3}=0$. Depending on the elastic constrain at the top extremity of the sensor, two different models can be obtained. 


\subsubsection{Asymptotic expansions of boundary conditions}

Elastic boundary condition at $x_{3}=h$. The elastic boundary condition considered in this analysis corresponds to the case where the displacement vanishes at $x_{3}=h$, i.e. the sensor is assumed to be clamped. Then the corresponding boundary condition reads

$$
\boldsymbol{T}_{0}(h, t)=\mathbf{0}, \quad r_{0}(h, t)=0, \quad t>0 .
$$

Remark 4.2 Otherwise, when a traction-free surface is assumed at $x_{3}=h$, one has at the order $\mathcal{O}(\delta)$

$$
\left(\mathcal{C}: \varepsilon_{S}\left[\boldsymbol{u}_{1}\right]+\mathbf{d} \cdot \nabla_{S} \varphi_{1}\right) \cdot \boldsymbol{n}+\left(\mathcal{C}: \varepsilon_{3}\left[\boldsymbol{u}_{0}\right]+\mathbf{d} \cdot \boldsymbol{e}_{3} \partial_{x_{3}} \varphi_{0}\right) \cdot \boldsymbol{n}=\mathbf{0}, \quad \hat{\boldsymbol{x}} \in \partial S \times\{h\}, \quad t>0 .
$$

Using that $\boldsymbol{n}=\boldsymbol{e}_{3}$, taking into account the expressions of $\boldsymbol{u}_{1}$ and $\varphi_{1}$ from $\sqrt{267}$, and multiplying the previous equation by the basis functions of $\mathcal{E}_{S, 0}$, then we obtain after integration the following relations

$$
\begin{cases}\overline{\mathbf{C}}_{\boldsymbol{t} t} \cdot \partial_{x_{3}} \boldsymbol{T}_{0}+\overline{\mathbf{c}}_{\boldsymbol{t} r} \partial_{x_{3}} r_{0}+\overline{\mathbf{d}}_{\boldsymbol{t} \phi} \partial_{x_{3}} \phi_{0}=\mathbf{0}, & \hat{\boldsymbol{x}} \in \partial S \times\{h\}, \quad t>0, \\ \overline{\mathbf{c}}_{r \boldsymbol{t}}^{\top} \cdot \partial_{x_{3}} \boldsymbol{T}_{0}+\overline{\mathrm{c}}_{r r} \partial_{x_{3}} r_{0}+\overline{\mathrm{d}}_{r \phi} \partial_{x_{3}} \phi_{0}=0, & \hat{\boldsymbol{x}} \in \partial S \times\{h\}, \quad t>0 .\end{cases}
$$

Elastic boundary condition at $x_{3}=0$ and well prepared data. At the bottom extremity of the sensor we have formally considered an imposed displacement field (10) characterized by (11), so that one has at the first order

$$
\boldsymbol{u}_{0}\left(x_{3}=0\right)=\boldsymbol{t}_{b}(t)+r_{b}(t) \boldsymbol{m}, \quad t>0 .
$$

However, we have extended to the whole domain the structure (23) of the displacement field obtained in the cross-section by formal asymptotic analysis. Moreover, depending on the nature of the boundary condition imposed on the cross-section, which is characterized by the subset $\Gamma_{0} \subset \partial S$, some simplifications occur in the resulting 1D model as shown in Section 4.2. Therefore, the above relation might not be meaningful in general and thus, we have to make additional assumptions on $\boldsymbol{t}_{b}$ to ensure its compatibility with the asymptotics (23). Classically, such assumptions amount to consider well-prepared data for the limit problem. Naturally we set

$$
r_{0}(0, t)=r_{b}(t), \quad t>0,
$$

moreover, for each of the configurations considered in Section 4.2, the elastic boundary condition at $x_{3}=0$ is substituted by a compatible one, i.e. we assume the existence of a time-dependent displacement vector $\boldsymbol{T}_{b}=\left[t_{b, 1} \ldots t_{b, d}\right]^{\top}$ such that

$$
\boldsymbol{T}_{0}(0, t)=\boldsymbol{T}_{b}(t), \quad t>0
$$

and which is characterized by the following properties

o If $\left|\Gamma_{0}\right|=0$ then $\boldsymbol{T}_{b} \in \mathbb{R}^{3}$ and $t_{b, 1}=t_{b, 2}=0$.

o When $\left|\Gamma_{0}\right| \neq 0$ and $\hat{\boldsymbol{x}} \cdot \boldsymbol{e}_{i}$ is constant on $\Gamma_{0}$ for $i=1$ or $i=2$ then $\boldsymbol{T}_{b} \in \mathbb{R}^{2}$ with $t_{b, i}=0$.

o If $\left|\Gamma_{0}\right| \neq 0$ with $\hat{\boldsymbol{x}} \cdot \boldsymbol{e}_{i}$ non-constant on $\Gamma_{0}$ for $i=1$ and 2 , then $\boldsymbol{T}_{b} \in \mathbb{R}^{2}$.

Of course these assumptions are restrictive and the definition of the vector $\boldsymbol{T}_{b}$ might seem artificial since such a discussion do not arise when dealing with the full 3D problem. In fact, if $\boldsymbol{t}_{b}$ does not satisfy the above conditions one can show then it is necessary to add a decaying term, or boundary layer, to the first order solution $\left(\varphi_{0}, \boldsymbol{u}_{0}\right)$ in order to obtain the correct asymptotics. However, the study of such boundary layer is beyond the scope of this article. 
Electric boundary conditions. Taking into account the boundary condition 112 and the expansion (19), we find at $x_{3}=0$ :

$$
\phi_{0}\left(x_{3}=0\right)=0, \quad t>0 .
$$

Moreover, the boundary condition governing the electric potential at $x_{3}=h$ depends on the user-chosen scaling of the resistance, namely the choice of the exponent $p$. On using the change of variables (15), the equation (13) reduces to

$\varphi\left(x_{3}=h\right)=-\delta^{1-p} R_{0} \frac{d}{d t} \int_{S, x_{3}=h}\left(\boldsymbol{\epsilon} \cdot \nabla_{S} \varphi-\mathbf{d}^{\top}: \varepsilon_{S}[\boldsymbol{u}]\right) \cdot \boldsymbol{n} \mathrm{d} \hat{s}-\delta^{2-p} R_{0} \frac{d}{d t} \int_{S, x_{3}=h}\left(\boldsymbol{\epsilon} \cdot \boldsymbol{e}_{3} \partial_{x_{3}} \varphi-\mathbf{d}^{\top}: \varepsilon_{3}[\boldsymbol{u}]\right) \cdot \boldsymbol{n} \mathrm{d} \hat{s}$,

Therefore, we can distinguish three cases leading to meaningful models relevant for the applications considered in this work:

o $p=2$. In this case, by identifying the $\mathcal{O}(1)$ terms in (54) one obtains

$$
\varphi_{0}\left(x_{3}=h\right)=-R_{0} \frac{d}{d t} \int_{S, x_{3}=h}\left(\boldsymbol{\epsilon} \cdot \boldsymbol{e}_{3} \partial_{x_{3}} \varphi_{0}-\mathbf{d}^{\top}: \varepsilon_{3}\left[\boldsymbol{u}_{0}\right]\right) \cdot \boldsymbol{n} \mathrm{d} \hat{s}-R_{0} \frac{d}{d t} \int_{S, x_{3}=h}\left(\boldsymbol{\epsilon} \cdot \nabla_{S} \varphi_{1}-\mathbf{d}^{\top}: \varepsilon_{S}\left[\boldsymbol{u}_{1}\right]\right) \cdot \boldsymbol{n} \mathrm{d} \hat{s},
$$

On noting that $\boldsymbol{n}=\boldsymbol{e}_{3}$ on the boundary at $x_{3}=h$ and taking into account the structure of $\left(\varphi_{0}, \mathbf{u}_{0}\right)$ given by (23), the definition (26) of $\left(\varphi_{1}, \boldsymbol{u}_{1}\right)$ and the definition of the homogenized coefficients in Section 4 then one can show that the boundary condition can be rewritten as

$$
\phi_{0}\left(x_{3}=h\right)=-R_{0} \frac{d}{d t}\left(\bar{\epsilon} \partial_{x_{3}} \phi_{0}-\overline{\mathbf{d}}_{\boldsymbol{t}}^{\top} \cdot \partial_{x_{3}} \boldsymbol{T}_{0}-\overline{\mathrm{d}}_{r} \partial_{x_{3}} r_{0}\right)_{x_{3}=h} .
$$

o $p=1$. In such a case, using the same arguments as above and identifying the $\mathcal{O}(1)$ terms we find that

$$
\phi_{0}\left(x_{3}=h\right)=-R_{0} \frac{d}{d t} \int_{S, x_{3}=h}\left(\boldsymbol{\epsilon} \cdot \nabla_{S} \varphi_{0}-\mathbf{d}^{\top}: \varepsilon_{S}\left[\boldsymbol{u}_{0}\right]\right) \cdot \boldsymbol{n} \mathrm{d} \hat{s}=0 .
$$

Next, the identification at order $\mathcal{O}(\delta)$ yields

$$
\varphi_{1}\left(x_{3}=h\right)=-R_{0} \frac{d}{d t} \int_{S, x_{3}=h}\left(\boldsymbol{\epsilon} \cdot \boldsymbol{e}_{3} \partial_{x_{3}} \varphi_{0}-\mathbf{d}^{\top}: \varepsilon_{3}\left[\boldsymbol{u}_{0}\right]\right) \cdot \boldsymbol{n} \mathrm{d} \hat{s}-R_{0} \frac{d}{d t} \int_{S, x_{3}=h}\left(\boldsymbol{\epsilon} \cdot \nabla_{S} \varphi_{1}-\mathbf{d}^{\top}: \varepsilon_{S}\left[\boldsymbol{u}_{1}\right]\right) \cdot \boldsymbol{n} \mathrm{d} \hat{s} .
$$

As for the case $p=2$, the previous relation can be simplified so that the boundary condition reads

$$
\varphi_{1}\left(x_{3}=h\right)=-R_{0} \frac{d}{d t}\left(\bar{\epsilon} \partial_{x_{3}} \phi_{0}-\overline{\mathbf{d}}_{\boldsymbol{t}}^{\top} \cdot \partial_{x_{3}} \boldsymbol{T}_{0}-\overline{\mathrm{d}}_{r} \partial_{x_{3}} r_{0}\right)_{x_{3}=h} .
$$

o $\underline{p=0}$. At the orders $\mathcal{O}(1)$ and $\mathcal{O}(\delta)$ we immediately obtain the relations

$$
\phi_{0}\left(x_{3}=h\right)=0 \quad \text { and } \quad \varphi_{1}\left(x_{3}=h\right)=-R_{0} \frac{d}{d t} \int_{S, x_{3}=h}\left(\boldsymbol{\epsilon} \cdot \nabla_{S} \varphi_{0}-\mathbf{d}^{\top}: \varepsilon_{S}\left[\boldsymbol{u}_{0}\right]\right) \cdot \boldsymbol{n} \mathrm{d} \hat{s}=0 .
$$

At the next order $\mathcal{O}\left(\delta^{2}\right)$, after simplifications one has

$$
\varphi_{2}\left(x_{3}=h\right)=-R_{0} \frac{d}{d t}\left(\bar{\epsilon} \partial_{x_{3}} \phi_{0}-\overline{\mathbf{d}}_{\boldsymbol{t}}^{\top} \cdot \partial_{x_{3}} \boldsymbol{T}_{0}-\overline{\mathrm{d}}_{r} \partial_{x_{3}} r_{0}\right)_{x_{3}=h} .
$$


The analysis of these different cases clearly shows that the chosen scaling of the resistance determines the order at which the homogenized electrical displacement, i.e. the term $-\bar{\epsilon} \partial_{x_{3}} \phi_{0}+\overline{\mathbf{d}}_{t}^{\top} \cdot \partial_{x_{3}} \boldsymbol{T}_{0}+\overline{\mathrm{d}}_{r} \partial_{x_{3}} r_{0}$, will contribute to the measured electric potential. The configuration with $p=2$ corresponds to the upper bound compatible with the asymptotic expansion 19 and decreasing $p$ shifts the available informations provided by the measurements to contributions at higher order (in particular compare the cases $p=1$ and $p=2$ ).

Remark 4.3 In the case where the piezoelectric bar is used as an actuator, i.e. when it is connected to an electric generator, then an $\mathcal{O}(1)$ source term $V(t)$ has to be added to the equation (54). In such a case, this term appears as a additional contribution to the first-order potential $\phi_{0}$ in the three cases considered above.

\subsubsection{Clamped sensor models}

We consider that the condition $(50)$ holds and we introduce the intermediate unknowns $\widetilde{\boldsymbol{T}}_{0}, \tilde{r}_{0}$ and $\tilde{\phi}_{0}$ which are functions of the space-time variables $\left(x_{3}, t\right)$, and satisfy the homogeneous boundary conditions

$$
\widetilde{\boldsymbol{T}}_{0}(0, t)=\widetilde{\boldsymbol{T}}_{0}(h, t)=\mathbf{0}, \quad \tilde{r}_{0}(0, t)=\tilde{r}_{0}(h, t)=0, \quad \tilde{\phi}_{0}(0, t)=\tilde{\phi}_{0}(h, t)=0, \quad t>0 .
$$

Then, the original set of unknowns $\boldsymbol{T}_{0}, r_{0}$ and $\phi_{0}$ of the 1D models can be decomposed as

$$
\begin{gathered}
\boldsymbol{T}_{0}\left(x_{3}, t\right)=\widetilde{\boldsymbol{T}}_{0}\left(x_{3}, t\right)+\left(1-\frac{x_{3}}{h}\right) \boldsymbol{T}_{b}(t), \quad r_{0}\left(x_{3}, t\right)=\tilde{r}_{0}\left(x_{3}, t\right)+\left(1-\frac{x_{3}}{h}\right) r_{b}(t), \\
\phi_{0}\left(x_{3}, t\right)=\tilde{\phi}_{0}\left(x_{3}, t\right)+\frac{x_{3}}{h} \phi_{t}(t),
\end{gathered}
$$

In the next paragraphs, we provide the equation satisfied by the function $\phi_{t}$, and we derive the associated complete $1 \mathrm{D}$ sensor model.

First, from the initial condition (18) we have

$$
\phi_{t}(0)=0
$$

Moreover, the equation on $\phi_{t}(t)$ can be obtained in a straightforward manner. Obviously, from the discussion in Section 4.3.1, this function depends strongly on the choice adopted for the scaling of the electrical resistance. Therefore, for the different cases considered we have

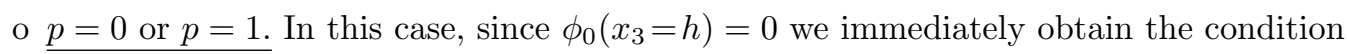

$$
\phi_{t}(t)=0, \quad t>0 \text {. }
$$

In such configurations, it is therefore necessary to derive the model at the order $\mathcal{O}(\delta)$ or $\mathcal{O}\left(\delta^{2}\right)$ to obtain a model that takes into account the measurement of the elastic displacement imposed at $x_{3}=0$.

o $p=2$. The following equation on $\phi_{t}$ is derived from the first equation of (37), after differentiation with

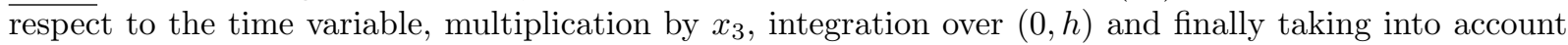
55):

$$
\bar{\epsilon} \frac{d}{d t} \phi_{t}(t)+\frac{h}{R_{0}} \phi_{t}(t)=-\overline{\mathbf{d}}_{\boldsymbol{t}}^{\top} \frac{d}{d t} \boldsymbol{T}_{b}-\bar{d}_{r} \frac{d}{d t} r_{b}, \quad t>0
$$

Next, the set of fields equations satisfied by the elastic unknowns can be derived.

Now, from the equations (37) and owing to the decomposition (59), the functions $\left(\widetilde{\boldsymbol{T}}_{0}, \tilde{r}_{0}, \tilde{\phi}_{0}\right)$ satisfy, for $t>0$ and $x_{3} \in(0, h)$

$$
\left\{\begin{array}{l}
\bar{\epsilon} \partial_{x_{3}}^{2} \tilde{\phi}_{0}-\overline{\mathbf{d}}_{\boldsymbol{t}}^{\top} \cdot \partial_{x_{3}}^{2} \widetilde{\boldsymbol{T}}_{0}-\overline{\mathrm{d}}_{r} \partial_{x_{3}}^{2} \tilde{r}_{0}=0 \\
\bar{\rho}_{\boldsymbol{t}} \partial_{t}^{2} \widetilde{\boldsymbol{T}}_{0}-\overline{\mathbf{C}}_{\boldsymbol{t} \boldsymbol{t}} \cdot \partial_{x_{3}}^{2} \widetilde{\boldsymbol{T}}_{0}-\overline{\mathbf{c}}_{\boldsymbol{t} r} \partial_{x_{3}}^{2} \tilde{r}_{0}-\overline{\mathbf{d}}_{\boldsymbol{t}} \partial_{x_{3}}^{2} \tilde{\phi}_{0}=-\bar{\rho}_{\boldsymbol{t}}\left(1-\frac{x_{3}}{h}\right) \partial_{t}^{2} \boldsymbol{T}_{b}, \\
\bar{\rho}_{r} \partial_{t}^{2} \tilde{r}_{0}-\overline{\mathbf{c}}_{r \boldsymbol{t}}^{\top} \cdot \partial_{x_{3}}^{2} \widetilde{\boldsymbol{T}}_{0}-\overline{\mathbf{c}}_{r r} \partial_{x_{3}}^{2} \tilde{r}_{0}-\overline{\mathrm{d}}_{r} \partial_{x_{3}}^{2} \tilde{\phi}_{0}=-\bar{\rho}_{r}\left(1-\frac{x_{3}}{h}\right) \partial_{t}^{2} r_{b} .
\end{array}\right.
$$


Taking into account the properties of the homogenized parameters and replacing the electric term using the first equation, one can finally obtain

$$
\left\{\begin{array}{l}
\bar{\rho}_{\boldsymbol{t}} \partial_{t}^{2} \widetilde{\boldsymbol{T}}_{0}-\left(\overline{\mathbf{C}}_{\boldsymbol{t} \boldsymbol{t}}+\frac{\overline{\mathbf{d}}_{\boldsymbol{t}} \overline{\mathbf{d}}_{\boldsymbol{t}}^{\top}}{\bar{\epsilon}}\right) \cdot \partial_{x_{3}}^{2} \widetilde{\boldsymbol{T}}_{0}-\left(\overline{\mathbf{c}}_{\boldsymbol{t} r}+\frac{\overline{\mathbf{d}}_{\boldsymbol{t}} \overline{\mathrm{d}}_{r}}{\bar{\epsilon}}\right) \partial_{x_{3}}^{2} \tilde{r}_{0}=-\bar{\rho}_{\boldsymbol{t}}\left(1-\frac{x_{3}}{h}\right) \partial_{t}^{2} \boldsymbol{T}_{b}, \\
\bar{\rho}_{r} \partial_{t}^{2} \tilde{r}_{0}-\left(\overline{\mathbf{c}}_{r \boldsymbol{t}}^{\top}+\frac{\overline{\mathrm{d}}_{r} \overline{\mathbf{d}}_{\boldsymbol{t}}^{\top}}{\bar{\epsilon}}\right) \cdot \partial_{x_{3}}^{2} \widetilde{\boldsymbol{T}}_{0}-\left(\overline{\mathbf{c}}_{r r}+\frac{\overline{\mathrm{d}}_{r}^{2}}{\bar{\epsilon}}\right) \partial_{x_{3}}^{2} \tilde{r}_{0}=-\bar{\rho}_{r}\left(1-\frac{x_{3}}{h}\right) \partial_{t}^{2} r_{b},
\end{array}\right.
$$

which is completed by the constrains (58) and the initial conditions

$$
\widetilde{\boldsymbol{T}}_{0}(t=0)=\partial_{t} \widetilde{\boldsymbol{T}}_{0}(t=0)=\mathbf{0}, \quad \tilde{r}_{0}(t=0)=\partial_{t} \tilde{r}_{0}(t=0)=0 .
$$

The above set of equations shows that the elastic unknowns can be decoupled from the electric potential. Note that even in the configuration of a piezoelectric actuator with an externally imposed electric potential, the previous equations could also be used to show that the elastic terms decouple from the associated electric source. For every fixed $t>0$, the electric potential $\tilde{\phi}_{0}$ can be recovered by solving the homogeneous Dirichlet problem

$$
\left\{\begin{array}{l}
\bar{\epsilon} \partial_{x_{3}}^{2} \tilde{\phi}_{0}(\cdot, t)=\overline{\mathbf{d}}_{t}^{\top} \cdot \partial_{x_{3}}^{2} \widetilde{\boldsymbol{T}}_{0}(\cdot, t)+\overline{\mathrm{d}}_{r} \partial_{x_{3}}^{2} \tilde{r}_{0}(\cdot, t), \quad x_{3} \in(0, h) \\
\tilde{\phi}_{0}(0, t)=\tilde{\phi}_{0}(h, t)=0 .
\end{array}\right.
$$

We will show in Section 5.2 that this clamped sensor model corresponds to a measurement-mode configuration, therefore particularly suited for the identification of the boundary displacement $\boldsymbol{T}_{b}$.

Remark 4.4 Alternatively, if we consider that a traction-free constrain at $x_{3}=h$ holds, then the original unknowns $\left(\boldsymbol{T}_{0}, r_{0}, \phi_{0}\right)$ have to be decomposed according to

$$
\begin{gathered}
\boldsymbol{T}_{0}\left(x_{3}, t\right)=\widetilde{\boldsymbol{T}}_{0}\left(x_{3}, t\right)+\frac{x_{3}}{h} \boldsymbol{T}_{t}(t)+\left(1-\frac{x_{3}}{h}\right) \boldsymbol{T}_{b}(t), \quad r_{0}\left(x_{3}, t\right)=\tilde{r}_{0}\left(x_{3}, t\right)+\frac{x_{3}}{h} r_{t}(t)+\left(1-\frac{x_{3}}{h}\right) r_{b}(t), \\
\phi_{0}\left(x_{3}, t\right)=\tilde{\phi}_{0}\left(x_{3}, t\right)+\frac{x_{3}}{h} \phi_{t}(t) .
\end{gathered}
$$

Now, a new set of coupled equations satisfied by $\left(\widetilde{\boldsymbol{T}}_{0}, \tilde{r}_{0}, \tilde{\phi}_{0}\right)$ as well as by the time-dependent functions $\boldsymbol{T}_{t}, r_{t}$ and $\phi_{t}$ have to be determined to obtain a complete $1 D$ model. Without deriving explicitly these equations, which turn out to be non-local in space, one can check that the potential $\phi_{t}$ satisfies the initial condition (60), as well as (61) when $p \in\{0,1\}$. When $p=2$, the equation (62) has to be substituted by

$$
\bar{\epsilon} \frac{d}{d t} \phi_{t}(t)+\frac{h}{R_{0}} \phi_{t}(t)=\overline{\mathbf{d}}_{t}^{\top} \frac{d}{d t}\left(\boldsymbol{T}_{t}-\boldsymbol{T}_{b}\right)+\bar{d}_{r} \frac{d}{d t}\left(r_{t}-r_{b}\right), \quad t>0
$$

The previous equation provides an example of the coupling existing between the unknowns of the $1 D$ problem, which therefore highlights the reason why such boundary condition has not been chosen to derive the limit model to be suitable to applications to inverse problems. In fact, such traction-free sensor model can be associated to an emission-mode configuration.

\section{Discussions}

\subsection{Comparison with Bernoulli-Navier rod models}


As pointed out in Introduction, there exists a variety of models that have been derived depending on the assumptions on the scaling of the data. In particular one can refer to [28, 8 for the case of inhomogeneous elastic rods and plates, as well as 29, 17] for the derivation of static 1D models of inhomogeneous piezoelectric rods. In this studies, the resulting models are classically derived using the formal asymptotic analysis framework based on the full 3D elastic or piezoelectric equations, and the obtained limit displacement fields are of BernoulliNavier type (or Kirchhoff-Love for plates). However, such results differ from the models derived in Section 4 and this section is concerned with a discussion of the discrepancy between them. In fact, one shows how the chosen scaling of the density $\rho$ completely modifies the structure of the solution.

For the sake of simplicity, let us assume that $\varphi_{0}\left(x_{3}=h\right)=0$, which corresponds to the case $p=1$ or $p=2$, and $\boldsymbol{u}_{0}\left(x_{3}=0\right)=\mathbf{0}$. Therefore, let us denote $\tilde{\varphi}_{0}$ and $\tilde{\boldsymbol{u}}_{0}$, the first-order terms associated to these hypotheses in order to underline that the following comparison involves the homogeneous part of the limit fields. Note that from Section 4.3 .2 , one has in this case $\phi_{0} \equiv \tilde{\phi}_{0}, r_{0} \equiv \tilde{r}_{0}$ and $\boldsymbol{T}_{0} \equiv \widetilde{\boldsymbol{T}}_{0}$.

The difference between the two models can be characterized by looking at the energy identity associated with the present asymptotic model. This relation is obtained by taking the time derivative of $(35)-(i)$ and then multiplying it by $\tilde{\varphi}_{0}$, while the second equation of 35 is multiplied by $\partial_{t} \tilde{\boldsymbol{u}}_{0}$. After integration over $\Omega$ and summation one obtains

$$
\begin{aligned}
\frac{1}{2} \frac{d}{d t} \int_{\Omega} \rho\left|\partial_{t} \tilde{\boldsymbol{u}}_{0}\right|^{2} \mathrm{~d} \hat{\boldsymbol{x}}+\int_{\Omega}\left(\varepsilon_{S}\left[\boldsymbol{u}_{1}\right]+\varepsilon_{3}\left[\tilde{\boldsymbol{u}}_{0}\right]\right) & : \mathcal{C}: \varepsilon_{3}\left[\partial_{t} \tilde{\boldsymbol{u}}_{0}\right] \mathrm{d} \hat{\boldsymbol{x}}+\int_{\Omega}\left(\nabla_{S} \partial_{t} \varphi_{1}+\boldsymbol{e}_{3} \partial_{x_{3}} \partial_{t} \tilde{\varphi}_{0}\right) \cdot \boldsymbol{\epsilon} \cdot \boldsymbol{e}_{3} \partial_{x_{3}} \tilde{\varphi}_{0} \mathrm{~d} \hat{\boldsymbol{x}} \\
& +\int_{\Omega}\left(\mathbf{d} \cdot \nabla_{S} \varphi_{1}: \varepsilon_{3}\left[\partial_{t} \tilde{\boldsymbol{u}}_{0}\right]-\mathbf{d}^{\top}: \boldsymbol{\varepsilon}_{S}\left[\partial_{t} \boldsymbol{u}_{1}\right] \cdot \boldsymbol{e}_{3} \partial_{x_{3}} \tilde{\varphi}_{0}\right) \mathrm{d} \hat{\boldsymbol{x}}=0
\end{aligned}
$$

Moreover, a similar calculation for (24) yields the identity

$$
\begin{array}{r}
\int_{\Omega}\left(\mathbf{d} \cdot \nabla_{S} \varphi_{1}: \varepsilon_{3}\left[\partial_{t} \tilde{\boldsymbol{u}}_{0}\right]-\mathbf{d}^{\top}: \varepsilon_{S}\left[\partial_{t} \boldsymbol{u}_{1}\right] \cdot \boldsymbol{e}_{3} \partial_{x_{3}} \tilde{\varphi}_{0}\right) \mathrm{d} \hat{\boldsymbol{x}}=\int_{\Omega}\left(\varepsilon_{S}\left[\boldsymbol{u}_{1}\right]+\varepsilon_{3}\left[\tilde{\boldsymbol{u}}_{0}\right]\right): \mathcal{C}: \varepsilon_{S}\left[\partial_{t} \boldsymbol{u}_{1}\right] \mathrm{d} \hat{\boldsymbol{x}} \\
+\int_{\Omega}\left(\nabla_{S} \partial_{t} \varphi_{1}+\boldsymbol{e}_{3} \partial_{x_{3}} \partial_{t} \tilde{\varphi}_{0}\right) \cdot \boldsymbol{\epsilon} \cdot \nabla_{S} \varphi_{1} \mathrm{~d} \hat{\boldsymbol{x}}
\end{array}
$$

Finally, combining (63) and 64), integrating in time between 0 and $t$ and using the homogenous initial condition, we get

$$
\begin{aligned}
\int_{\Omega} \rho\left|\partial_{t} \tilde{\boldsymbol{u}}_{0}\right|^{2} \mathrm{~d} \hat{\boldsymbol{x}}+\int_{\Omega}\left(\varepsilon_{S}\left[\boldsymbol{u}_{1}\right]+\varepsilon_{3}\left[\tilde{\boldsymbol{u}}_{0}\right]\right): \mathcal{C}:\left(\varepsilon_{S}\left[\boldsymbol{u}_{1}\right]+\varepsilon_{3}\left[\tilde{\boldsymbol{u}}_{0}\right]\right) \mathrm{d} \hat{\boldsymbol{x}}+ \\
\qquad \int_{\Omega}\left(\nabla_{S} \varphi_{1}+\boldsymbol{e}_{3} \partial_{x_{3}} \tilde{\varphi}_{0}\right) \cdot \boldsymbol{\epsilon} \cdot\left(\nabla_{S} \varphi_{1}+\boldsymbol{e}_{3} \partial_{x_{3}} \tilde{\varphi}_{0}\right) \mathrm{d} \hat{\boldsymbol{x}}=0 .
\end{aligned}
$$

Based again on the initial conditions $(14)$, this energy identity yields $\tilde{\boldsymbol{u}}_{0}=\mathbf{0}$, as expected for the first-order contribution. However, if we assume either one of the following scalings in the asymptotic analysis

$$
\rho^{\delta}=\delta^{2} \rho \circ \mathcal{G}_{\delta}^{-1} \quad \text { or } \quad \partial_{t}^{2} \rightarrow \delta^{2} \partial_{t}^{2}
$$

i.e. either a scaling of the mass density or a scaling of the time variable that empathizes the long time behavior, then after formal asymptotic expansion using the ansazt $\sqrt{19}$, , the equation (24) remains valid, together with (35) having set $\rho=0$ formally. Therefore, the energy identity 65 still holds with $\rho=0$ as well, so that we finally obtain

$$
\varepsilon_{S}\left[\boldsymbol{u}_{1}\right]+\varepsilon_{3}\left[\tilde{\boldsymbol{u}}_{0}\right]=\mathbf{0} \quad \text { and } \quad \boldsymbol{\nabla}_{S} \varphi_{1}+\boldsymbol{e}_{3} \partial_{x_{3}} \tilde{\varphi}_{0}=\mathbf{0} \quad \text { in } \Omega .
$$

As we have already shown that $\nabla_{S} \varphi_{0}=\mathbf{0}$ in $\Omega$, the previous equation entails $\partial_{x_{3}} \varphi_{0}=0$ and therefore $\varphi_{0}=0$ using the homogenous Dirichlet boundary conditions, and $\varphi_{1}(\hat{\boldsymbol{x}}, t)=\phi_{1}\left(x_{3}, t\right)$ which means that there is no 
electric field at the order $\mathcal{O}(1)$. Similarly, since $\varepsilon_{S}\left[\tilde{\boldsymbol{u}}_{0}\right]=\mathbf{0}$ in $\Omega$ we also deduce from the first equation of 66 that $\tilde{\boldsymbol{u}}_{0}+\delta \boldsymbol{u}_{1}$ is of Benouilli-Navier type, i.e.

$$
\begin{aligned}
& \tilde{\boldsymbol{u}}_{0}(\hat{\boldsymbol{x}}, t) \equiv t_{0,1}\left(x_{3}, t\right) \boldsymbol{e}_{1}+t_{0,2}\left(x_{3}, t\right) \boldsymbol{e}_{2} \\
& \boldsymbol{u}_{1}(\hat{\boldsymbol{x}}, t) \equiv \boldsymbol{t}_{1}\left(x_{3}, t\right)-\left(\hat{x}_{1} \partial_{x_{3}} t_{0,1}\left(x_{3}, t\right)+\hat{x}_{2} \partial_{x_{3}} t_{0,2}\left(x_{3}, t\right)\right) \boldsymbol{e}_{3}+\boldsymbol{m}\left(\hat{x}_{1}, \hat{x}_{2}\right) r_{1}\left(x_{3}, t\right)
\end{aligned}
$$

in term of the scalar and vector functions $r_{1}$ and $\boldsymbol{t}_{1}$ respectively. Finally, if the boundary conditions or the source terms are scaled appropriately with respect to $\delta$, then the limit solution of the piezoelectric problem is not trivial and has the structure given by the previous equation.

\subsection{Coupling with probed medium and boundary displacement identification}

We consider now the application to non-destructive testing for which the piezoelectric bar is used as a passive sensor. We denote by $\Omega_{s}$ the underlying elastic solid, i.e. the probed body, in contact with the piezoelectric bar $\Omega^{\delta}$. The boundaries of the two domains intersect only on $\Sigma^{\delta}=\delta S \times\{0\}$ (see Figure 1). In the probed medium the displacement field $\boldsymbol{u}_{s}^{\delta}$ satisfies the classical elastodynamic equation

$$
\rho_{s} \partial_{t}^{2} \boldsymbol{u}_{s}^{\delta}-\operatorname{div} \mathcal{C}_{s}: \varepsilon\left[\boldsymbol{u}_{s}^{\delta}\right]=\boldsymbol{f}, \quad \boldsymbol{x} \in \Omega_{s}, \quad t>0
$$

where $f$ is an external source term. This equation is completed with the free-surface boundary condition

$$
\mathcal{C}_{s}: \varepsilon\left[\boldsymbol{u}_{s}^{\delta}\right] \cdot \boldsymbol{n}=\mathbf{0}, \quad \boldsymbol{x} \in \partial \Omega_{s} \backslash \Sigma^{\delta}, \quad t>0,
$$

together with the coupling conditions

$$
\boldsymbol{u}_{s}^{\delta}=\boldsymbol{u}^{\delta} \quad \text { and } \quad \mathcal{C}_{s}: \varepsilon\left[\boldsymbol{u}_{s}^{\delta}\right] \cdot \boldsymbol{e}_{3}=\left(\mathcal{C}^{\delta}: \varepsilon\left[\boldsymbol{u}^{\delta}\right]+\mathbf{d}^{\delta} \cdot \nabla \varphi^{\delta}\right) \cdot \boldsymbol{e}_{3}, \quad \boldsymbol{x} \in \Sigma^{\delta}, \quad t>0 .
$$

Note that the dependance in $\delta$, indicated by the corresponding superscript, of the solution inside $\Omega_{s}$ arises from the boundary condition, i.e. it is a consequence of the coupling with $\boldsymbol{u}^{\delta}$. The function $\boldsymbol{u}^{\delta}$ is defined by the piezoelectric equations $(6)$ as well as by boundary conditions $(7,9),(12)$ and $(13)$. However, the coupling conditions (67) is now substituted to the condition 107 .

Now, we formally extend the result given in [14] for the Helmholtz equation, to the case of transient elasticity. Doing so we conjecture a number of results whose proofs are postponed to future work.

Remark 5.1 Note that the main difficulty here lies in the time-dependent nature of the junction problem between the piezoelectric rod and the underlying elastic body. Moreover, the boundary layers that might be generated inside the piezoelectric bar due to the coupling with the limit field associated with $\boldsymbol{u}_{s}$ have to be analyzed in detail.

We conjecture the following limits

$$
\boldsymbol{u}_{s}^{\delta} \underset{\delta \rightarrow 0}{\longrightarrow} \boldsymbol{u}_{s, 0} \quad \text { and } \quad \boldsymbol{u}^{\delta} \underset{\delta \rightarrow 0}{\longrightarrow} \boldsymbol{u}_{0} \circ \mathcal{G}_{\delta}^{-1}
$$

with the convergence occurring in a sense to be discussed and where $\boldsymbol{u}_{s, 0}$ is defined as the solution of

$$
\begin{cases}\rho_{s} \partial_{t}^{2} \boldsymbol{u}_{s, 0}-\operatorname{div} \mathcal{C}_{s}: \varepsilon\left[\boldsymbol{u}_{s, 0}\right]=\boldsymbol{f}, & \boldsymbol{x} \in \Omega_{s}, \quad t>0 \\ \mathcal{C}_{s}: \boldsymbol{\varepsilon}\left[\boldsymbol{u}_{s, 0}\right] \cdot \boldsymbol{n}=\mathbf{0}, & \boldsymbol{x} \in \partial \Omega_{s}, \quad t>0\end{cases}
$$

Moreover, one has $\boldsymbol{u}_{0}=\boldsymbol{t}_{0}+r_{0} \boldsymbol{m}$ with $\boldsymbol{T}_{0}:=\left[t_{0,1} \ldots t_{0, d}\right]^{\top}$ and $\left(\boldsymbol{T}_{0}, r_{0}\right)$ is solution of (37) with appropriate boundary conditions. For the electric potential either one of the equation (55), (56) or (57) holds at $x_{3}=h$ and 
(53) at $x_{3}=0$. Regarding the elastic displacement, the condition (50) is satisfied at $x_{3}=h$. Lastly, at $x_{3}=0$ one considers, as in (51) and (52), that for $t>0$

$$
\begin{aligned}
& r_{0}(0, t)=r_{b}(t):=\lim _{\delta \rightarrow 0}\left|\Sigma^{\delta}\right|^{-1} \int_{\Sigma^{\delta}} \boldsymbol{u}_{s, 0} \cdot\left(\boldsymbol{m} \circ \mathcal{G}_{\delta}^{-1}\right) \mathrm{d} s^{\delta}, \\
& \boldsymbol{T}_{0}(0, t)=\boldsymbol{T}_{b}(t),
\end{aligned}
$$

where $\boldsymbol{T}_{b}(t)$ is defined hereafter depending on the nature of the constrain on $\partial S^{\delta}$. Following the discussion in Section 4.3.1 concerning the well-preparedness of the data, one has

o If $\left|\Gamma_{0}\right|=0$ then $\boldsymbol{T}_{b} \in \mathbb{R}^{3}$ and $t_{b, 1}=t_{b, 2}=0$ while

$$
t_{3}:=\lim _{\delta \rightarrow 0}\left|\Sigma^{\delta}\right|^{-1} \int_{\Sigma^{\delta}} \boldsymbol{u}_{s, 0} \cdot \boldsymbol{e}_{3} \mathrm{~d} s^{\delta} .
$$

o When $\left|\Gamma_{0}\right| \neq 0$ and $\hat{\boldsymbol{x}} \cdot \boldsymbol{e}_{i}$ is constant on $\Gamma_{0}$ for $i=1$ or $i=2$ then $\boldsymbol{T}_{b} \in \mathbb{R}^{2}$ with $t_{b, i}=0$ and

$$
t_{b, j}:=\lim _{\delta \rightarrow 0}\left|\Sigma^{\delta}\right|^{-1} \int_{\Sigma^{\delta}} \boldsymbol{u}_{s, 0} \cdot \boldsymbol{e}_{j} \mathrm{~d} s^{\delta}, \quad j \in\{1,2\}, j \neq i .
$$

o If $\left|\Gamma_{0}\right| \neq 0$ with $\hat{\boldsymbol{x}} \cdot \boldsymbol{e}_{i}$ non-constant on $\Gamma_{0}$ for $i=1$ and 2 , then $\boldsymbol{T}_{b} \in \mathbb{R}^{2}$ with

$$
t_{b, i}:=\lim _{\delta \rightarrow 0}\left|\Sigma^{\delta}\right|^{-1} \int_{\Sigma^{\delta}} \boldsymbol{u}_{s, 0} \cdot \boldsymbol{e}_{i} \mathrm{~d} s^{\delta}, \quad i \in\{1,2\} .
$$

In other words, we conjecture that the well-preparedness of the data naturally arise at the first order from the asymptotic analysis of the full junction problem. The components of $\boldsymbol{u}_{s, 0}$ that do not enter the 1D limit piezoelectric problem generates boundary layers that vanishes, in a sense to be determined, in the limit $\delta \rightarrow 0$.

Remark 5.2 If $\rho$ is constant and assuming enough regularity on $\boldsymbol{u}_{s, 0}$, then by formal Taylor expansion of $\boldsymbol{u}_{s, 0}$ in the above definition of $r_{0}$ and owing to (5), one can check that $r_{0}(0, t)=0$.

Now, in the context of non-destructive testing or inverse scattering problems, we address the question of the identification of the displacement field $\left.\boldsymbol{u}_{s}^{\delta}\right|_{\Sigma^{\delta}}$ in the asymptotics $\delta \rightarrow 0$, i.e. that of the reconstruction of the term $\boldsymbol{T}_{b}$ defined above. We want to determine the quantity $\boldsymbol{T}_{b}$ as a function of time from the measurements which are provided by the electric potential recorded at the top of the sensor, i.e. we assume that

$$
\varphi^{\delta}\left(x_{3}=h, t\right)=\phi_{0}(h, t)+\delta \varphi_{1}\left(x_{3}=h, t\right)+\ldots \text { is known. }
$$

We denote $q(t)$ the electric charge flowing at time $t$, through an electrode connected to the piezoelectric bar at $\delta S \times\{h\}$. Therefore one has

$$
q(t)=\left(\bar{\epsilon} \partial_{x_{3}} \phi_{0}-\overline{\mathbf{d}}_{\boldsymbol{t}}^{\top} \cdot \partial_{x_{3}} \boldsymbol{T}_{0}-\overline{\mathrm{d}}_{r} \partial_{x_{3}} r_{0}\right)(h, t) .
$$

From the results in Section 4.3.1 we have for the configuration considered with $p \in\{0,1,2\}$

$$
\varphi^{\delta}\left(x_{3}=h, t\right)=-\delta^{2-p} R_{0} \frac{d}{d t} q(t)+\mathcal{O}\left(\delta^{3-p}\right),
$$


which means that the quantity $q(t)$ is known, after integration in time, up to $\mathcal{O}(\delta)$ additional contributions. By integrating twice the equation (37)-(i) with respect to $x_{3}$, we find that

$$
q(t)=\frac{1}{h}\left(\bar{\epsilon} \phi_{0}(h, t)+\overline{\mathbf{d}}_{\boldsymbol{t}}^{\top} \cdot \boldsymbol{T}_{b}(t)+\overline{\mathrm{d}}_{r} r_{b}(t)\right) .
$$

This equation shows how the measured electric charge is expressed in terms of the boundary displacement $\overline{\mathbf{d}}_{\boldsymbol{t}}^{\mathrm{T}} \cdot \boldsymbol{T}_{b}+\overline{\mathrm{d}}_{r} r_{b}$. This quantity is therefore related to the structure of the limit problem, in particular to the definition of $\Gamma_{0}$, as well as to the homogenized coefficients $\overline{\mathbf{d}}_{\boldsymbol{t}}$ and $\overline{\mathrm{d}}_{r}$.

For example, assuming for the sake of simplicity that $r_{b}(t)=0$, e.g. when $\rho$ is constant as in Remark 5.2 . and $p \in\{0,1\}$ which entails $\phi_{0}(h, t)=0$, then one has the simple relation

$$
q(t)=h^{-1} \overline{\mathbf{d}}_{\boldsymbol{t}}^{\top} \cdot \boldsymbol{T}_{b}(t)
$$

which enables to directly recover the boundary displacement $\boldsymbol{T}_{b}$ up to the scalar product with $\overline{\mathbf{d}}_{\boldsymbol{t}}$. This last equation directly justifies the type of boundary conditions considered in this analysis which therefore allow a simple interpretation of the measurements. Moreover, in the case $d=2$, one has access to the transverse components of the displacement, which is not possible when $d=3$. Finally, when choosing $p=2$, the problem can be tackle in a similar fashion after solving the scalar ordinary differential equation 62 and treating the problem as presented in Section 4.3.2.

\section{Conclusion}

In this study we have addressed the question of determining the asymptotic dynamical behavior of passive piezoelectric bars with respect to their small transverse dimensions. Based on the 3D quasi-static piezoelectric model and using formal asymptotic expansions, the structure of the limit electric and displacement fields are obtained at the first order. Moreover, the corresponding limit dynamical equations with the associated homogenized material parameters have been obtained and analyzed. A number of boundary conditions imposed on the lateral surface of the bar have been considered in order to show that different limit models can arise asymptotically, and in particular that the transverse components of the displacement may, or may not, enter the resulting equations. Given the assumptions considered, these conclusions are general since the boundary conditions at the extremities of the bar have not been introduced at this point and only the original interior field equations have been analyzed.

Next, these results are particularized to the application considered where the piezoelectric bar is employed as a passive sensor providing an electric measurement of the boundary displacement field associated with an underlying solid illuminated by an external source. In this configuration, the electric and elastic boundary conditions at the top and bottom extremities of the bar have been specified and corresponding limit problems have been finally obtained. In particular, it is shown that there exists a configuration for which the interior equation satisfied by the displacement field is decoupled from the electric potential, the latter satisfying an ordinary differential equations with the source term expressed in terms of the former. Finally, in the asymptotic limit, the measured electric charge is shown to be directly expressed as a linear combination of the components of the displacement at the interface with the probed medium. Such result is of key importance for the inverse problem considered and therefore it justifies a posteriori the choice of imposed boundary conditions in the original 3D problem.

Theoretical work remains to be done to provide convergence results and error estimates associated with the limit electric and displacement fields. The dynamical junction problem of the piezoelectric bar with the underlying body requires deeper mathematical analysis for a rigorous justification of the equations 10 and (11). Such an analysis would allow to discuss the existence and properties of boundary layers that are needed to derive complete error estimates. Finally, from a practical and computational standpoints, it appears to be necessary to assess the obtained limit models from the perspective of the inverse problem. These avenues will be pursued in the future. 


\section{References}

[1] G. Allaire and M. Amar. Boundary layer tails in periodic homogenization. ESAIM. Control, Optimisation and Calculus of Variations. European Series in Applied and Industrial Mathematics, 4:209-243 (electronic), 1999.

[2] L. Alvarez-Vazquez and J. M. Viaño. Asymptotic justification of an evolution linear thermoelastic model for rods. Comput. Methods Appl. Mech. Engrg, 115:93-109, 1994.

[3] T. Arens. Linear sampling methods for 2D inverse elastic wave scattering. Inverse Problems, 17:1445-1464, 2001.

[4] C. Bellis and M. Bonnet. A FEM-based topological sensitivity approach for fast qualitative identification of buried cavities from elastodynamic overdetermined boundary data. International Journal of Solids and Structures, 47(9):1221-1242, 2010.

[5] M. Bonnet. Topological sensitivity for 3D elastodynamic and acoustic inverse scattering in the time domain. Comp. Meth. Appl. Mech. Engng., 195:5239-5254, 2006.

[6] M. Bonnet and B. B. Guzina. Elastic-wave identification of penetrable obstacles using shape-material sensitivity framework. J. Comp. Phys., 228:294-311, 2009.

[7] A. Charalambopoulos, A. Kirsch, K. A. Anagnostopoulos, D. Gintides, and K. Kiriaki. The factorization method in inverse elastic scattering from penetrable bodies. Inverse Problems, 23:27-51, 2007.

[8] P. G. Ciarlet. Mathematical Elasticity, Vol. II: Theory of plates. Studies in Mathematics and its Applications. North-Holland, Amsterdam, 1997.

[9] N. Dominguez, V. Gibiat, and Y. Esquerre. Time domain topological gradient and time reversal analogy: an inverse method for ultrasonic target detection. Wave Motion, 42(1):31-52, 2005.

[10] K. O. Friedrichs and R. F. Dressler. A boundary-layer theory for elastic plates. Communications on Pure and Applied Mathematics, 14:1-33, 1961.

[11] T. Ikeda. Fundamentals of piezoelectricity. Oxford University Press, 1990.

[12] S. Imperiale and P. Joly. Mathematical and numerical modelling of piezoelectric sensors. ESAIM: Mathematical Modelling and Numerical Analysis, 46:875-909, 2012.

[13] H. Irago and J. M. Viaño. Mathematical justification of stretching and torsional vibration models for elastic rods. Comput. Methods Appl. Mech. Engrg, 189:975-989, 2000.

[14] P. Joly and S. Tordeux. Asymptotic analysis of an approximate model for time harmonic waves in media with thin slots. M2AN Math. Model. Numer. Anal., 40:63-97, 2006.

[15] G. A. Maugin and D. Attou. An asymptotic theory of thin piezoelectric plates. Q. J. Mech. Appl. Math., 43:347-362, 1990.

[16] I. M. Narra Figueiredo and C. M. Franco Leal. A piezoelectric anisotropic plate model. Asymptotic Analysis, 44:327-346, 2005.

[17] I. M. Narra Figueiredo and C. M. Franco Leal. A generalized piezoelectric Bernoulli-Navier anisotropic rod model. J. Elasticity, 85:85-106, 2006.

[18] S. A. Nazarov. Justification of the asymptotic theory of thin rods. Integral and pointwise estimates. $J$. Math. Sci., 97:4245-4279, 1999. 
[19] S. Nintcheu Fata and B. B. Guzina. A linear sampling method for near-field inverse problems in elastodynamics. Inverse Problems, 20:713-736, 2004.

[20] S. Nintcheu Fata, B. B. Guzina, and M. Bonnet. Computational framework for the BIE solution to inverse scattering problems in elastodynamics. Computational Mechanics, 32:370-380, 2003.

[21] R. E. Plessix, Y. H. De Roeck, and G. Chavent. Waveform inversion of reflection seismic data for kinematic parameters by local optimization. SIAM J. Sci. Comput., 20:1033-1052, 1999.

[22] M. Rahmoune, A. Benjeddou, and R. Ohayon. New thin piezoelectric plate models. J. Intell. Mater. Syst. Struct., 9:1017-1029, 1998.

[23] I. T. Rekanos, T. V. Yioultsis, and T. D. Tsiboukis. Inverse scattering using the finite element method and a nonlinear optimization technique. IEEE Transactions on microwave theory and techniques, 47:336-344, 1999 .

[24] D. Royer and E. Dieulesaint. Elastic waves in solids I. Free and guided propagation. Springer, 2000.

[25] D. Royer and E. Dieulesaint. Elastic waves in solids II. Generation, Acousto-optic interaction, Applications. Springer, 2000.

[26] L. Schmerr Jr and S.J. Song. Ultrasonic nondestructive evaluation systems. Springer, 2007.

[27] A. Sene. Modelling of piezoelectric static thin plates. Asymptotic Analysis, 25:1-20, 2001.

[28] L. Trabucho and J. M. Viaño. Mathematical modelling of rods. In P. G. Ciarlet and J. L. Lions, editors, Handbook of Numerical Analysis, volume 4 of Studies in Mathematics and its Applications, pages $487-974$. North-Holland, Amsterdam, 1996.

[29] T. Weller and C. Licht. Asymptotic modeling of linearly piezoelectric slender rods. C. R. Mecanique, 336:572-577, 2008. 